\title{
Dynamics of a General Stochastic Nonautonomous Lotka-Volterra Model with Delays and Impulsive Perturbations
}

\author{
Qing Wang, Yongguang Yu, and Shuo Zhang \\ Department of Mathematics, Beijing Jiaotong University, Beijing 100044, China \\ Correspondence should be addressed to Yongguang Yu; ygyu@bjtu.edu.cn
}

Received 23 May 2015; Revised 9 October 2015; Accepted 11 October 2015

Academic Editor: Ming Mei

Copyright (C) 2015 Qing Wang et al. This is an open access article distributed under the Creative Commons Attribution License, which permits unrestricted use, distribution, and reproduction in any medium, provided the original work is properly cited.

\begin{abstract}
A stochastic nonautonomous $\mathrm{N}$-species Lotka-Volterra model with delays and impulsive perturbations is investigated. For this model, sufficient conditions for extinction, nonpersistence in the mean, weak persistence and stochastic permanence are given, respectively. The influences of the stochastic noises, and the impulsive perturbations on the properties of the stochastic model are also discussed. The critical value between weak persistence and extinction is obtained. Finally, numerical simulations are given to support the theoretical analysis results.
\end{abstract}

\section{Introduction}

Population ecology is a major subfield of ecology that deals with the dynamics of species populations and the way these populations interact with the environment. It is concerned with the study of groups of organisms that live together in time and space and compete for the limited resources or in some way inhibit others' growth. Modelling of dynamic interactions in nature allows us to understand better how these complex interactions and processes work. The wellknown model that regards dynamic of population models is the Lotka-Volterra model. The investigation of the LotkaVolterra model is one of the dominant themes in mathematical ecology due to its importance. The Lotka-Volterra model with delays has received more and more attentions and had lots of nice results [1-5]. More details of the Lotka-Volterra model with delays are discussed in the books by Gopalsamy [6] and Kuang [7].

In the real world, the population models are inevitably influenced by the environmental noise which is an important component in an ecosystem [8-10]. Moreover, May [11] has pointed out the fact that, due to environmental noise, the birth rate, carrying capacity, competition coefficient, and other parameters involved with the system exhibit random fluctuation to a greater or lesser extent [12].
On the other hand, populations may be affected by a variety of factors both naturally and manly, such as earthquake, drought, flooding, fire, crop-dusting, planting, hunting, and harvesting; the inner discipline of species or environment often suffers some dispersed changes over a relatively short time interval at the fixed times, which makes it unsuitable to be considered continually. In mathematics perspective, such sudden changes could be described by impulses. With the development of the theory of impulsive differential equations $[13,14]$, we can establish adequate mathematical models of impulsive differential equations to investigate the dynamic behaviors of such ecosystems with impulsive effects. Consequently the dynamical behaviors of impulsive population dynamical models and stochastic population dynamical models have been extensively studied [15-19]. The nonautonomous $\mathrm{N}$-species Lotka-Volterra competitive system with impulsive perturbations was discussed in Hou et al. [15]. Nspecies nonautonomous Lotka-Volterra competitive system with delays and impulsive perturbations was considered in Zhang and Teng [18]. While these papers did not discuss the persistence and extinction of the stochastic Lotka-Volterra model, from the viewpoint of applications, it is critical to find out when the population will go to extinction or survival.

A major problem in population biology is to understand what determines extinction of a population. Population 
extinction is often a result of habitat destruction and modification which can be widespread. Moreover, dramatic changes in ecosystem structure or function are often caused by the species additions in the form of invasive species. In addition, the extinction of native populations may be caused by the growth of invasive species [20]. Moreover, even large populations may be destroyed by some extraordinary perturbation [21]. When the time is sufficiently large the population of some species may not become extinct, but the size of that population may be close to zero so that the species can be endangered. In other words, there exists a critical number between extinction and survival of population. In this sense, Ma and Hallam [22, 23] proposed the concepts of nonpersistence in the mean and weak persistence for some deterministic models and Lu and Ding [24] applied these concepts to stochastic logistic models instead of the stochastic Lotka-Volterra model.

Inspired by the works referred above, in this paper, we will investigate the persistence and extinction of a general stochastic nonautonomous Lotka-Volterra model with delays and impulsive perturbations. To our knowledge, there are few results of this aspect for the stochastic nonautonomous Lotka-Volterra model. Moreover, all the publications have not obtained the persistence-extinction threshold for the general stochastic nonautonomous Lotka-Volterra model with delays and impulsive perturbations. The problems above are explored and some main results are given in this paper. The general stochastic nonautonomous Lotka-Volterra model with delays and impulsive perturbations which has a unique positive global solution is investigated. For this model, sufficient conditions for extinction, nonpersistence in the mean, weak persistence, and stochastic permanence are established. The influences of the stochastic noises and impulsive perturbations on the properties of the stochastic model are discussed. Comparing with deterministic results [25-27], if the noise is sufficiently small, the property permanence that the related deterministic system possesses is preserved in the stochastic model. However, with the increase of stochastic noise, the properties of the system may be changed greatly. For example, the solution to the associated stochastic model will be extinct with probability one with the increase of stochastic noise being sufficiently large, although the solution to the original deterministic model may be persistent. The properties of the system are not affected by the impulsive perturbations which are bounded; otherwise, the properties may be changed significantly. The critical value between weak persistence and extinction is obtained.

The rest of the paper is arranged as follows. The stochastic nonautonomous Lotka-Volterra model with delays and impulsive perturbations is formulated and some notations and preliminaries are given in Section 2. Section 3 shows that the general nonautonomous Lotka-Volterra model has a unique positive global solution. Then, sufficient conditions for extinction, nonpersistence in the mean, weak persistence, and stochastic permanence are established in Section 4 . The simulation results in Section 5 are given to illustrate the main results obtained in this paper. Finally, the conclusions are given in Section 6.

\section{Problem Formulation and Preliminaries}

A classical nonautonomous Lotka-Volterra model with timevarying and infinite delays can be expressed as follows:

$$
\begin{aligned}
& \frac{d x_{i}(t)}{d t}=x_{i}(t)\left[r_{i}(t)-\sum_{j=1}^{n} a_{i j}(t) x_{j}(t)\right. \\
& +\sum_{j=1}^{n} b_{i j}(t) x_{j}\left(t-\tau_{i j}(t)\right) \\
& \left.+\sum_{j=1}^{n} c_{i j}(t) \int_{-\infty}^{0} x_{j}(t+\theta) d \mu_{i j}(\theta)\right], \\
& \quad 1 \leq i \leq n, 1 \leq j \leq n,
\end{aligned}
$$

where $x(t)=\left(x_{1}(t), \ldots, x_{n}(t)\right)^{T} ; x_{i}(t)$ and $r_{i}(t)$ are the population size and intrinsic exponential growth rate for the $i$ th species at time $t$, respectively; $a_{i j}(t), b_{i j}(t)$, and $c_{i j}(t)$ represent the effects of interspecific (for $i \neq j$ ) and intraspecific (for $i=$ $j$ ) interaction at time $t ; \tau_{i j}(t) \geq 0$ represents the time-varying delays; and $\mu_{i j}(\theta)$ is the probability measure on $(-\infty, 0], 1 \leq$ $i, j \leq n$.

In real life, model (1) is affected by environmental noises. The intrinsic growth rate of the $i$ th species $r_{i}(t)(1 \leq i \leq$ $n$ ) at time $t$ is estimated by an average value plus an error term. Then the intrinsic growth rate becomes $r_{i}(t) \rightarrow$ $r_{i}(t)+\sigma_{i}(t) \dot{B}_{i}(t)$. The effects of interspecific (for $i \neq j$ ) and intraspecific (for $i=j$ ) interaction $a_{i j}(t)(1 \leq i, j \leq n$ ) at time $t$ are estimated by an average value plus an error term. So $a_{i j}(t)$ is replaced by $-a_{i j}(t) \rightarrow-a_{i j}(t)+\delta_{i j}(t) \dot{B}_{i j}(t) . \sigma_{i}(t)$ and $\delta_{i j}(t)(1 \leq i, j \leq n)$ are continuous nonnegative bounded functions on $\bar{R}_{+}=[0,+\infty)$ and the intensities of the white noise at time $t . \dot{B}_{i}(t)$ and $\dot{B}_{i j}(t)$ are the white noises; $B_{i}(t)$ and $B_{i j}(t)$ are the one-dimensional Brownian motions defined on a complete probability space $(\Omega, \mathscr{F}, \mathscr{P})$.

On the other hand, in practice, model (1) is affected by impulsive perturbations. As a result, model (1) becomes the following stochastic nonautonomous Lotka-Volterra model with impulsive perturbations:

$$
\begin{aligned}
& d x_{i}(t)=x_{i}(t)\left[r_{i}(t)-\sum_{j=1}^{n} a_{i j}(t) x_{j}(t)\right. \\
& +\sum_{j=1}^{n} b_{i j}(t) x_{j}\left(t-\tau_{i j}(t)\right) \\
& \left.+\sum_{j=1}^{n} c_{i j}(t) \int_{-\infty}^{0} x_{j}(t+\theta) d \mu_{i j}(\theta)\right] d t+x_{i}(t) \\
& \cdot \sigma_{i}(t) d B_{i}(t)+x_{i}(t) \sum_{j=1}^{n} \delta_{i j}(t) x_{j}(t) d B_{i j}(t), \\
& x_{i}\left(t_{k}^{+}\right)-x_{i}\left(t_{k}\right)=h_{i k} x_{i}\left(t_{k}\right), \quad t \neq t_{k}, \quad k \in N, \\
& k \in N, 1 \leq i \leq n, 1 \leq j \leq n,
\end{aligned}
$$


where $N$ denotes the set of positive integers, $0<t_{1}<t_{2}<$ $\cdots<\lim _{k \rightarrow+\infty} t_{k}=+\infty$. This model will be studied in this paper.

Let the initial data $\xi(\theta)=\left(\xi_{1}(\theta), \xi_{2}(\theta), \ldots, \xi_{n}(\theta)\right)^{T}$ be positive and belong to the friendly space $\mathscr{C}_{g}$ [28] which is defined by

$$
\begin{aligned}
\mathscr{C}_{g} & =\left\{\varphi \in C\left((-\infty, 0] ;(0,+\infty)^{n}\right): l<\|\varphi\|_{\mathscr{C}_{g}}\right. \\
& \left.=\sup _{-\infty<\theta \leq 0} e^{r \theta}|\varphi(\theta)|<L\right\},
\end{aligned}
$$

where $|\varphi(\theta)|=\left(\varphi_{1}^{2}(\theta)+\varphi_{2}^{2}(\theta)+\cdots+\varphi_{n}^{2}(\theta)\right)^{1 / 2}, r>0$, and $l, L$ are two positive constants, $l<L$.

For model (2) we always assume the following:

(H1): $\mu_{i j}(\theta)(1 \leq i, j \leq n)$ is the probability measure on $(-\infty, 0]$ satisfying $\mu_{i j r}=\int_{-\infty}^{0} e^{-2 r \theta} d \mu_{i j}(\theta)<+\infty$.

(H2): $r_{i}(t), a_{i j}(t), b_{i j}(t)$ and $c_{i j}(t)(1 \leq i, j \leq n)$ are continuous and bounded function on $\bar{R}_{+}$and $\min _{1 \leq i, j \leq n} \inf _{t \in \bar{R}_{+}} a_{i j}(t)>0, b_{i j}(t) \geq 0, c_{i j}(t) \geq 0$.

(H3): $\tau_{i j}(t)(1 \leq i, j \leq n)$ are continuously differentiable functions with $0 \leq \tau_{i j}(t) \leq \tau^{M}$ and $1-\dot{\tau}_{i j}(t)>0$ for $t \in R$, where $\tau^{M}$ is a constant. $\Delta_{i j}^{-1}(t)$ is inverse function of $\Delta_{i j}(t)=t-\tau_{i j}(t)$.

(H4): we assume that $1+h_{i k}>0, i=1,2, \ldots, n, k \in N$. When $h_{i k}>0$, the perturbation denotes increasing of the species (e.g., planting), while $-1<h_{i k}<0$ represents decreasing (e.g., harvesting).

Definition 1 (see [29]). A stochastic process $X(t)=\left(X_{1}(t)\right.$, $\left.\ldots, X_{n}(t)\right)^{T}, t \in \bar{R}^{+}$, is said to be a solution of ISDE:

$$
\begin{gathered}
d X(t)=F(t, X(t)) d t+G(t, X(t)) d B(t), \\
t \neq t_{k}, k \in N, \\
X\left(t_{k}^{+}\right)-X\left(t_{k}\right)=B_{k} X\left(t_{k}\right), \quad k \in N
\end{gathered}
$$

with initial condition $X(0)$, if the following conditions are satisfied:

(1) $X(t)$ is adapted and is continuous on $\left(0, t_{1}\right)$ and $\left(t_{k}, t_{k+1}\right), k \in N ; F(t, X(t)) \in L^{1}\left(\bar{R}_{+} ; R^{n}\right), G(t, X(t)) \epsilon$ $L^{2}\left(\bar{R}_{+} ; R^{n}\right)$, where $L^{k}\left(\bar{R}_{+} ; R^{n}\right)$ is all $R^{n}$ valued measurable adapted processes $f(t)$ satisfying $\int_{0}^{T}|f(t)|^{k} d t<$ $+\infty$ a.s. (almost surely) for every $T>0$.

(2) For each $t_{k}, k \in N, X\left(t_{k}^{+}\right)=\lim _{t \rightarrow t_{k}^{+}} X(t)$ and $X\left(t_{k}^{-}\right)=\lim _{t \rightarrow t_{k}^{-}} X(t)$ exist and $X\left(t_{k}\right)=X\left(t_{k}^{-}\right)$with probability one.

(3) $X(t)$ obeys the equivalent integral equation of (4) for almost every $t \in \bar{R}_{+} \backslash t_{k}$ and satisfies the impulsive conditions at each $t=t_{k}, k \in N$ with probability one. Then $X(t)$ is said to be a solution of ISDE (4).
For the aim of simplicity, we define the following notations:

$$
\begin{aligned}
f^{u} & =\sup _{t \in R} f(t), \\
f^{l} & =\inf _{t \in R} f(t), \\
\left\langle x_{i}(t)\right\rangle=\frac{1}{t} \int_{0}^{t} x_{i}(s) d s, & \\
x_{i}^{*} & =\limsup _{t \rightarrow+\infty} x_{i}(t), \\
x_{i^{*}} & =\liminf _{t \rightarrow+\infty} x_{i}(t), \\
R_{+} & =(0,+\infty), \\
g_{i}^{*} & =\limsup _{t \rightarrow+\infty} t^{-1}\left[\sum_{0<t_{k}<t} \ln \left(1+h_{i k}\right)\right. \\
& \left.+\int_{0}^{t}\left(r_{i}(t)-\frac{\sigma_{i}^{2}(s)}{2}\right) d s\right], \quad 1 \leq i \leq n .
\end{aligned}
$$

For any sequence $\left\{d_{i j}(t)\right\}(1 \leq i, j \leq n)$, define

$$
\begin{aligned}
& \left(\bar{d}_{i j}^{u}\right)=\max _{1 \leq i, j \leq n} \sup _{t \in R} d_{i j}(t), \\
& \left(\tilde{d}_{i j}^{l}\right)=\min _{1 \leq i, j \leq n} \inf _{t \in R} d_{i j}(t) .
\end{aligned}
$$

The following definitions are commonly used and we list them here.

Definition 2. (a) The population $x_{i}(t)$ is said to go to extinction a.s. if $\lim _{t \rightarrow+\infty} x_{i}(t)=0$.

(b) The population $x_{i}(t)$ is said to be nonpersistence in the mean a.s. (see, e.g., [22]) if $\lim \sup _{t \rightarrow+\infty}\left\langle x_{i}(t)\right\rangle=0$.

(c) The population $x_{i}(t)$ is said to be weak persistence a.s. (see, e.g., [23]) if $\lim \sup _{t \rightarrow+\infty} x_{i}(t)>0$.

(d) The population $x(t)=\left(x_{1}(t), x_{2}(t), \ldots, x_{n}(t)\right)^{T}$ is said to be stochastic permanence if, for arbitrary $\varepsilon>0$, there are constants $\beta>0, H>0$ such that $\liminf _{t \rightarrow+\infty} \mathscr{P}\{|x(t)| \geq$ $\beta\} \geq 1-\varepsilon$ and $\liminf _{t \rightarrow+\infty} \mathscr{P}\{|x(t)| \leq H\} \geq 1-\varepsilon$, where $|\cdot|$ denotes the Euclidian norm in $R_{+}^{n}$.

\section{Nonexplosion}

Theorem 3. Consider model (2); for any given initial value $\xi(\theta)=\left(\xi_{1}(\theta), \xi_{2}(\theta), \ldots, \xi_{n}(\theta)\right)^{T} \in \mathscr{C}_{g}$, there is a unique solution $x(t)$ on $t \in R$ and the solution remains in $R_{+}^{n}$ with probability 1; in other words, $x(t) \in R_{+}^{n}$ for all $t \in R$ almost surely. 
Proof. Consider the following SDE without impulses:

$$
\begin{aligned}
& d y_{i}(t)=y_{i}(t)\left[r_{i}(t)-\sum_{j=1}^{n} a_{i j}(t) \prod_{0<t_{k}<t}\left(1+h_{j k}\right) y_{j}(t)\right. \\
& +\sum_{j=1}^{n} b_{i j}(t) \prod_{0<t_{k}<t-\tau_{i j}(t)}\left(1+h_{j k}\right) y_{j}\left(t-\tau_{i j}(t)\right) \\
& +\sum_{j=1}^{n} c_{i j}(t) \\
& \left.\cdot \int_{-\infty}^{0} \prod_{0<t_{k}<t+\theta}\left(1+h_{j k}\right) y_{j}(t+\theta) d \mu_{i j}(\theta)\right] d t \\
& +y_{i}(t) \sigma_{i}(t) d B_{i}(t)+y_{i}(t) \sum_{j=1}^{n} \delta_{i j}(t) \prod_{0<t_{k}<t}\left(1+h_{j k}\right) \\
& \cdot y_{j}(t) d B_{i j}(t),
\end{aligned}
$$

with the same initial condition as model (2).

Now for model (7), there is a unique solution $y(t)$ on $t \in R$ and the solution will remain in $R_{+}$with probability 1 .

Since the coefficients of model (7) do not fulfil the linear growth condition, the general theorems of existence and uniqueness cannot be implemented for this equation. However, they are locally Lipschitz continuous; hence for any given positive initial condition $\xi(\theta)=\left(\xi_{1}(\theta), \xi_{2}(\theta), \ldots\right.$, $\left.\xi_{n}(\theta)\right)^{T} \in \mathscr{C}_{g}$, there is a unique local solution $y(t)$ on $t \in$ $\left(-\infty, \tau_{e}\right)$, where $\tau_{e}$ is the explosion time. To show this solution $x(t)$ is global solution, which means that the explosion time $\tau_{e}=+\infty$, a.s. Let us choose $k_{0}>0$ which is sufficiently large such that

$$
\frac{1}{k_{0}}<\min _{-\infty<\theta \leq 0}|\xi(\theta)| \leq \max _{-\infty<\theta \leq 0}|\xi(\theta)|<k_{0} .
$$

For each integer $k \geq k_{0}$, define the stopping time

$$
\tau_{k}=\inf \left\{t \in\left(-\infty, \tau_{e}\right): y_{i}(t) \leq \frac{1}{k} \text { or } y_{i}(t) \geq k\right\},
$$

$$
1 \leq i \leq n \text {, }
$$

where throughout this paper we set inf $\varnothing=+\infty$ (as usual $\varnothing$ denotes the empty set). Clearly, $\tau_{k}$ is increasing as $k \rightarrow+\infty$. Set $\tau_{+\infty}=\lim _{k \rightarrow+\infty} \tau_{k}$, whence $\tau_{+\infty} \leq \tau_{e}$ a.s. and $x(t) \in R_{+}^{n}$ a.s. for all $t \geq 0$. In other words, to complete the proof all we need to show is that $\tau_{+\infty}=+\infty$ a.s. To show this statement, let us define a $C^{2}$-function $V: R_{+}^{n} \rightarrow R_{+}$by $V_{1}(y)=\sum_{i=1}^{n}\left[\sqrt{y_{i}}-\right.$ $\left.1-0.5 \ln \left(y_{i}\right)\right]$, where $y=\left(y_{1}, \ldots, y_{n}\right)^{T}$. Let $k \geq k_{0}$ and $T>0$ be arbitrary. Now define

$$
\begin{aligned}
& V(t, y(t))=\sum_{i=1}^{n} \sum_{j=1}^{n} \int_{t-\tau_{i j}(t)}^{t} \frac{\left(\prod_{0<t_{k}<s}\left(1+h_{j k}\right)\right)^{2} y_{j}^{2}(s)}{1-\dot{\tau}_{i j}\left(\Delta_{i j}^{-1}(s)\right)} d s \\
& \quad+\sum_{i=1}^{n} \sum_{j=1}^{n} \int_{-\infty}^{0} \int_{t+\theta}^{t}\left(\prod_{0<t_{k}<s}\left(1+h_{j k}\right)\right)^{2} y_{j}^{2}(s) d s d \mu_{i j}(\theta) \\
& +V_{1}(y(t)) .
\end{aligned}
$$

For $0 \leq t \leq \tau_{k} \wedge T$, applying Itô's formula to $V(t, y(t))$, we can get

$$
\begin{gathered}
d V(t, y(t))=F(y(t)) d t+\sum_{i=1}^{n} \frac{1}{2}\left[y_{i}^{0.5}(t)-1\right] \\
\cdot \sigma_{i}(t) d B_{i}(t)+\sum_{i=1}^{n} \frac{1}{2}\left[y_{i}^{0.5}(t)-1\right] \\
\cdot\left(\sum_{j=1}^{n} \delta_{i j}(t) \prod_{0<t_{k}<t}\left(1+h_{j k}\right) y_{j}(t)\right) d B_{i j}(t),
\end{gathered}
$$

where

$$
\begin{aligned}
F(y(t)) & \sum_{i=1}^{n} \sum_{j=1}^{n} \frac{\left(\prod_{0<t_{k}<t}\left(1+h_{j k}\right)\right)^{2} y_{j}^{2}(t)}{1-\dot{t}_{i j}\left(\Delta_{i j}^{-1}(t)\right)} \\
& +n \sum_{j=1}^{n}\left(\prod_{0<t_{k}<t}\left(1+h_{j k}\right)\right)^{2} y_{j}^{2}(t) \\
& +\sum_{i=1}^{n} \frac{1}{2} r_{i}(t)\left[y_{i}^{0.5}(t)-1\right] \\
& -\sum_{i=1}^{n} \sum_{j=1}^{n} \frac{1}{2} a_{i j}(t)\left[y_{i}^{0.5}(t)-1\right] \prod_{0<t_{k}<t}\left(1+h_{j k}\right) y_{j}(t) \\
& +\sum_{i=1}^{n} \sum_{j=1}^{n} \frac{1}{16} b_{i j}^{2}(t)\left[y_{i}^{0.5}(t)-1\right]^{2} \\
& +\sum_{i=1}^{n} \sum_{j=1}^{n} \frac{1}{16} c_{i j}^{2}(t)\left[y_{i}^{0.5}(t)-1\right]^{2} \\
& +\frac{1}{8} \sum_{i=1}^{n} \sigma_{i}^{2}(t) y_{i}^{0.5}(t)+\frac{1}{4} \sum_{i=1}^{n} \sigma_{i}^{2}(t) \\
& -\frac{1}{8} \sum_{i=1}^{n} y_{i}^{0.5}(t)\left[\sum_{j=1}^{n} \delta_{i j}(t) \prod_{0<t_{k}<t}\left(1+h_{j k}\right) y_{j}(t)\right]^{2} \\
&
\end{aligned}
$$

With the fact that $\prod_{0<t_{k}<t}\left(1+h_{i k}\right) y_{i}(t) \leq \sum_{i=1}^{n} \prod_{0<t_{k}<t}(1+$ $\left.h_{i k}\right) y_{i}(t)$, it is easy to see that $F(y(t))$ is bounded on $R_{+}^{n}$. In other words, there exists a positive constant $K$ such that $F(y(t)) \leq K$. Therefore,

$$
\begin{aligned}
& d V(t, y(t)) \leq K d t+\sum_{i=1}^{n} \frac{1}{2}\left[y_{i}^{0.5}(t)-1\right] \sigma_{i}(t) d B_{i}(t) \\
& +\sum_{i=1}^{n} \frac{1}{2}\left[y_{i}^{0.5}(t)-1\right] \\
& \quad\left(\sum_{j=1}^{n} \delta_{i j}(t) \prod_{0<t_{k}<t}\left(1+h_{j k}\right) y_{j}(t)\right) d B_{i j}(t) .
\end{aligned}
$$


Integrating both sides from 0 to $t$ and then taking expectations, we have

$$
\begin{aligned}
& E V(t, y(t)) \\
& \quad \leq \sum_{i=1}^{n} \sum_{j=1}^{n} \int_{-\tau_{i j}(0)}^{0} \frac{\left(\prod_{0<t_{k}<t}\left(1+h_{j k}\right)\right)^{2} y_{j}^{2}(s)}{1-\dot{\tau}_{i j}\left(\Delta_{i j}^{-1}(s)\right)} d s \\
& \quad+\sum_{i=1}^{n} \sum_{j=1}^{n} \int_{-\infty}^{0} \int_{\theta}^{0}\left(\prod_{0<t_{k}<s}\left(1+h_{j k}\right)\right)^{2} y_{j}^{2}(s) d s d \mu_{i j}(\theta) \\
& +V_{1}(y(0))+K t .
\end{aligned}
$$

Let $t=\tau_{k} \wedge T$, from $\dot{\tau}_{i j}\left(\Delta_{i j}^{-1}(t)\right)<1$, we have

$$
\begin{aligned}
& E V_{1}\left(y\left(\tau_{k} \wedge T\right)\right) \\
& \quad \leq \sum_{i=1}^{n} \sum_{j=1}^{n} \int_{-\tau_{i j}(0)}^{0} \frac{\left(\prod_{0<t_{k}<t}\left(1+h_{j k}\right)\right)^{2} y_{j}^{2}(s)}{1-\dot{\tau}_{i j}\left(\Delta_{i j}^{-1}(s)\right)} d s \\
& \quad+\sum_{i=1}^{n} \sum_{j=1}^{n} \int_{-\infty}^{0} \int_{\theta}^{0}\left(\prod_{0<t_{k}<s}\left(1+h_{j k}\right)\right)^{2} y_{j}^{2}(s) d s d \mu_{i j}(\theta) \\
& +V_{1}(y(0))+K T .
\end{aligned}
$$

Note that, for every $\omega \in\left\{\tau_{k} \leq T\right\}, x_{i}\left(\tau_{k}, \omega\right)$ equals either $k$ or $1 / k$, and hence $V_{1}\left(y\left(\tau_{k}, \omega\right)\right)$ is no less than either $\sqrt{k}-1-$ $0.5 \log (k)$ or $\sqrt{1 / k}-1-0.5 \log (1 / k)=\sqrt{1 / k}-1+0.5 \log (k)$. Consequently,

$$
\begin{aligned}
V_{1}\left(y\left(\tau_{k}, \omega\right)\right) \geq & {[\sqrt{k}-1-0.5 \log (k)] } \\
& \wedge\left[\sqrt{\frac{1}{k}}-1+0.5 \log (k)\right] .
\end{aligned}
$$

It then follows from (14) that

$$
\begin{aligned}
& \sum_{i=1}^{n} \sum_{j=1}^{n} \int_{-\tau_{i j}(0)}^{0} \frac{\left(\prod_{0<t_{k}<t}\left(1+h_{j k}\right)\right)^{2} y_{j}^{2}(s)}{1-\dot{\tau}_{i j}\left(\Delta_{i j}^{-1}(s)\right)} d s \\
& \quad+\sum_{i=1}^{n} \sum_{j=1}^{n} \int_{-\infty}^{0} \int_{\theta}^{0}\left(\prod_{0<t_{k}<s}\left(1+h_{j k}\right)\right)^{2} y_{j}^{2}(s) d s d \mu_{i j}(\theta) \\
& \quad+V_{1}(y(0))+K T \geq E\left[I_{\left\{\tau_{k} \leq T\right\}}(\omega) V_{1}\left(y\left(\tau_{k}, \omega\right)\right)\right] \\
& \quad \geq \mathscr{P}\left\{\tau_{k} \leq T\right\}\left([ \sqrt { k } - 1 - 0 . 5 \operatorname { l o g } ( k ) ] \wedge \left[\sqrt{\frac{1}{k}}-1\right.\right. \\
& \quad+0.5 \log (k)]),
\end{aligned}
$$

where $I_{\left\{\tau_{k} \leq T\right\}}$ is the indicator function of $\left\{\tau_{k} \leq T\right\}$. Letting $k \rightarrow+\infty, \lim _{k \rightarrow+\infty} \mathscr{P}\left\{\tau_{k} \leq T\right\}=0$ and hence $\mathscr{P}\left\{\tau_{+\infty} \leq\right.$ $T\}=0$. Since $T>0$ is arbitrary, we must have $\mathscr{P}\left\{\tau_{+\infty}<\right.$ $+\infty\}=0$, so $\mathscr{P}\left\{\tau_{+\infty}=+\infty\right\}=1$ as required.
Now let

$$
x_{i}(t)=\prod_{0<t_{k}<t}\left(1+h_{i k}\right) y_{i}(t) .
$$

Next $x(t)$ is the solution of (2) which will be shown. In fact, $x(t)$ is continuous on $\left(t_{k}, t_{k+1}\right) \subset(0,+\infty), k \in N$ and for every $t \neq t_{k}$

$$
\begin{aligned}
& d x_{i}(t)=d\left[\prod_{0<t_{k}<t}\left(1+h_{i k}\right) y_{i}(t)\right] \\
& =\prod_{0<t_{k}<t}\left(1+h_{i k}\right) d y_{i}(t)=\prod_{0<t_{k}<t}\left(1+h_{i k}\right) \\
& \cdot y_{i}(t)\left[r_{i}(t)-\sum_{j=1}^{n} a_{i j}(t) \prod_{0<t_{k}<t}\left(1+h_{j k}\right) y_{j}(t)\right. \\
& +\sum_{j=1}^{n} b_{i j}(t) \prod_{0<t_{k}<t-\tau_{i j}(t)}\left(1+h_{j k}\right) y_{j}\left(t-\tau_{i j}(t)\right) \\
& +\sum_{j=1}^{n} c_{i j}(t) \\
& \left.\cdot \int_{-\infty}^{0} \prod_{0<t_{k}<t+\theta}\left(1+h_{j k}\right) y_{j}(t+\theta) d \mu_{i j}(\theta)\right] d t \\
& +\prod_{0<t_{k}<t}\left(1+h_{i k}\right) \sigma_{i}(t) y_{i}(t) d B_{i}(t) \\
& +\prod_{0<t_{k}<t}\left(1+h_{i k}\right) y_{i}(t) \sum_{j=1}^{n} \delta_{i j}(t) \prod_{0<t_{k}<t}\left(1+h_{j k}\right) \\
& y_{j}(t) d B_{i j}(t)=x_{i}(t)\left[r_{i}(t)\right. \\
& -\sum_{j=1}^{n} a_{i j}(t) x_{j}(t)+\sum_{j=1}^{n} b_{i j}(t) x_{j}\left(t-\tau_{i j}(t)\right) \\
& \left.+\sum_{j=1}^{n} c_{i j}(t) \int_{-\infty}^{0} x_{j}(t+\theta) d \mu_{i j}(\theta)\right] d t+\sigma_{i}(t) \\
& \cdot x_{i}(t) d B_{i}(t)+x_{i}(t) \sum_{j=1}^{n} \delta_{i j}(t) x_{j}(t) d B_{i j}(t) .
\end{aligned}
$$

Moreover, for every $k \in N$ and $t_{k} \in[0,+\infty)$,

$$
\begin{aligned}
x_{i}\left(t_{k}^{+}\right) & =\lim _{t \rightarrow t_{k}^{+}} \prod_{0<t_{l}<t}\left(1+h_{i l}\right) y_{i}(t) \\
& =\prod_{0<t_{l} \leq t_{k}}\left(1+h_{i l}\right) y_{i}\left(t_{k}^{+}\right) \\
& =\left(1+h_{i k}\right) \prod_{0<t_{l}<t_{k}}\left(1+h_{i l}\right) y_{i}\left(t_{k}\right) \\
& =\left(1+h_{i k}\right) x_{i}\left(t_{k}\right) .
\end{aligned}
$$


In addition,

$$
\begin{aligned}
& x_{i}\left(t_{k}^{-}\right) \\
& \quad=\lim _{t \rightarrow t_{k}^{-}} \prod_{0<t_{l}<t}\left(1+h_{i l}\right) y_{i}(t) \prod_{0<t_{l}<t_{k}}\left(1+h_{i l}\right) y_{i}\left(t_{k}^{-}\right) \\
& \quad=\prod_{0<t_{l}<t_{k}}\left(1+h_{i l}\right) y_{i}\left(t_{k}\right)=x_{i}\left(t_{k}\right) .
\end{aligned}
$$

So the proof is completed.

\section{The Persistence and Extinction Analysis}

In this section, the extinction, nonpersistence in the mean, weak persistence, and stochastic permanence of model (2) are discussed.

Theorem 4. If $g_{i}^{*}<0$ and $\min _{1 \leq j \leq n} \inf _{t \in \bar{R}_{+}}\left\{a_{i j}(t)-b_{i j}\left(\Delta_{i j}^{-1}(t)\right) /\right.$ $\left.\left(1-\dot{\tau}_{i j}\left(\Delta_{i j}^{-1}(t)\right)\right)-c_{i j}^{u}\right\} \geq 0$, then the ith population $x_{i}(t)$ of model (2) goes to extinction a.s.

Proof. Now applying Itô's formula to (7), we can have

$$
\begin{aligned}
d \sum_{j=1}^{n} \int_{t-\tau_{i j}(t)}^{t} \frac{b_{i j}\left(\Delta_{i j}^{-1}(s)\right) \prod_{0<t_{k}<s}\left(1+h_{j k}\right) y_{j}(s)}{1-\dot{\tau}_{i j}\left(\Delta_{i j}^{-1}(s)\right)} d s \\
+d \ln y_{i}(t) \\
\quad=\sum_{j=1}^{n}\left[\frac{b_{i j}\left(\Delta_{i j}^{-1}(t)\right) \prod_{0<t_{k}<t}\left(1+h_{j k}\right) y_{j}(t)}{1-\dot{\tau}_{i j}\left(\Delta_{i j}^{-1}(t)\right)}\right. \\
\left.+b_{i j}(t) \prod_{0<t_{k}<t-\tau_{i j}(t)}\left(1+h_{j k}\right) y_{j}\left(t-\tau_{i j}(t)\right)\right] d t \\
+\left[r_{i}(t)-\frac{\sigma_{i}^{2}(t)}{2}-\sum_{j=1}^{n} a_{i j}(t) \prod_{0<t_{k}<t}\left(1+h_{j k}\right) y_{j}(t)\right. \\
+\sigma_{i}(t) d B_{i}(t)+\sum_{j=1}^{n} \delta_{i j}(t) \prod_{0<t_{k}<t}\left(1+h_{j k}\right) \\
+\sum_{j=1}^{n} b_{i j}(t) \prod_{0<t_{k}<t-\tau_{i j}(t)}\left(1+h_{j k}\right) y_{j}\left(t-\tau_{i j}(t)\right) \\
+\sum_{j=1}^{n} c_{i j}(t) \int_{-\infty}^{0} \prod_{0<t_{k}<t+\theta}\left(1+h_{j k}\right) y_{j}(t+\theta) d \mu_{i j}(\theta) \\
\left.+\sum_{j=1}^{n} \delta_{i j}(t) \prod_{0<t_{k}<t}\left(1+h_{j k}\right) y_{j}(t)\right]^{2}(t) .
\end{aligned}
$$

The quadratic form of $M_{i}^{1}(t)$ is $\left\langle M_{i}^{1}(t), M_{i}^{1}(t)\right\rangle=\int_{0}^{t} \sigma_{i}^{2}(s) d s \leq$ $\left(\sigma_{i}^{u}\right)^{2} t$. Making use of the strong law of large numbers for martingales [30],

$$
\lim _{t \rightarrow+\infty} \frac{M_{i}^{1}(t)}{t}=0 \text {, a.s. }
$$

The quadratic form of $M_{i}^{2}(t)$ is $\left\langle M_{i}^{2}(t), M_{i}^{2}(t)\right\rangle=$ $\int_{0}^{t}\left[\sum_{j=1}^{n} \delta_{i j}(s) x_{j}(s)\right]^{2} d s$. By virtue of the exponential martingale inequality [30], for any positive constants $T_{0}, \alpha$ and $\beta$, we have

$$
\begin{gathered}
\mathscr{P}\left\{\sup _{0 \leq t \leq T_{0}}\left[M_{i}^{2}(t)-\frac{\alpha}{2}\left\langle M_{i}^{2}(t), M_{i}^{2}(t)\right\rangle\right]>\beta\right\} \\
\leq e^{-\alpha \beta} .
\end{gathered}
$$


Choose $T_{0}=k, \alpha=1, \beta=2 \ln k$. Then it follows that

$$
\begin{aligned}
& \mathscr{P}\left\{\sup _{0 \leq t \leq k}\left[M_{i}^{2}(t)-\frac{1}{2}\left\langle M_{i}^{2}(t), M_{i}^{2}(t)\right\rangle\right]>2 \ln k\right\} \\
& \leq \frac{1}{k^{2}} .
\end{aligned}
$$

Making use of the Borel-Cantelli lemma [30], one gets that, for almost all $\omega \in \Omega$, there is a random integer $k_{0}=k_{0}(\omega)$ such that, for $k \geq k_{0}$,

$$
\sup _{0 \leq t \leq k}\left[M_{i}^{2}(t)-\frac{1}{2}\left\langle M_{i}^{2}(t), M_{i}^{2}(t)\right\rangle\right] \leq 2 \ln k .
$$

This implies that

$$
\begin{aligned}
M_{i}^{2}(t) & \leq 2 \ln k+\frac{1}{2}\left\langle M_{i}^{2}(t), M_{i}^{2}(t)\right\rangle \\
& =2 \ln k+\frac{1}{2} \int_{0}^{t}\left[\sum_{j=1}^{n} \delta_{i j}(s) x_{j}(s)\right]^{2} d s
\end{aligned}
$$

for all $0 \leq t \leq k, k \geq k_{0}$ a.s. Substituting this inequality, (24), and (25) into (23), we can obtain that

$$
\begin{aligned}
& \sum_{0<t_{k}<t} \ln \left(1+h_{i k}\right)+\ln y_{i}(t)-\ln y_{i}(0) \leq \sum_{0<t_{k}<t} \ln (1 \\
& \left.+h_{i k}\right)+\sum_{j=1}^{n} \int_{-\tau_{i j}(0)}^{0}\left[\frac{b_{i j}\left(\Delta_{i j}^{-1}(s)\right) x_{j}(s)}{1-\dot{\tau}_{i j}\left(\Delta_{i j}^{-1}(s)\right)}\right] d s \\
& +\int_{0}^{t}\left[r_{i}(s)-\frac{\sigma_{i}^{2}(s)}{2}\right. \\
& -\sum_{j=1}^{n}\left(a_{i j}(s)-\frac{b_{i j}\left(\Delta_{i j}^{-1}(s)\right)}{1-\dot{\tau}_{i j}\left(\Delta_{i j}^{-1}(s)\right)}-c_{i j}^{u}\right) \\
& \left.\cdot x_{j}(s)\right] d s+2 \ln k+\sum_{j=1}^{n} \frac{1}{r} c_{i j}^{u}\left\|\xi_{j}\right\|_{\mathscr{C}_{r}} \mu_{i j r}(1 \\
& \left.-e^{-r t}\right)+M_{i}^{1}(t),
\end{aligned}
$$

for all $0 \leq t \leq k, k \geq k_{0}$ a.s. Therefore,

$$
\begin{aligned}
& \ln x_{i}(t)-\ln x_{i}(0) \leq \sum_{0<t_{k}<t} \ln \left(1+h_{i k}\right) \\
& +\sum_{j=1}^{n} \int_{-\tau_{i j}(0)}^{0}\left[\frac{b_{i j}\left(\Delta_{i j}^{-1}(s)\right) x_{j}(s)}{1-\dot{\tau}_{i j}\left(\Delta_{i j}^{-1}(s)\right)}\right] d s+\int_{0}^{t}\left[r_{i}(s)\right. \\
& -\frac{\sigma_{i}^{2}(s)}{2}-\sum_{j=1}^{n}\left(a_{i j}(s)-\frac{b_{i j}\left(\Delta_{i j}^{-1}(s)\right)}{1-\dot{\tau}_{i j}\left(\Delta_{i j}^{-1}(s)\right)}-c_{i j}^{u}\right) \\
& \left.\cdot x_{j}(s)\right] d s+2 \ln k+\sum_{j=1}^{n} \frac{1}{r} c_{i j}^{u}\left\|\xi_{j}\right\|_{\mathscr{C}_{r}} \mu_{i j r}(1 \\
& \left.-e^{-r t}\right)+M_{i}^{1}(t),
\end{aligned}
$$

for all $0 \leq t \leq k, k \geq k_{0}$ a.s. In other words, we can get that for $0<k-1 \leq t \leq k, k \geq k_{0}$,

$$
\begin{aligned}
& t^{-1}\left\{\ln x_{i}(t)-\ln x_{i}(0)\right\} \\
& \leq t^{-1} \sum_{0<t_{k}<t} \ln \left(1+h_{i k}\right) \\
& \quad+t^{-1} \sum_{j=1}^{n} \int_{-\tau_{i j}(0)}^{0}\left[\frac{b_{i j}\left(\Delta_{i j}^{-1}(s)\right) x_{j}(s)}{1-\dot{\tau}_{i j}\left(\Delta_{i j}^{-1}(s)\right)}\right] d s \\
& \quad+t^{-1} \int_{0}^{t}\left[r_{i}(s)-\frac{\sigma_{i}^{2}(s)}{2}\right] d s+2(k-1)^{-1} \ln k \\
& \quad+t^{-1} \sum_{j=1}^{n} \frac{1}{r} c_{i j}^{u}\left\|\xi_{j}\right\|_{\mathscr{C}_{r}} \mu_{i j r}\left(1-e^{-r t}\right)+\frac{M_{i}^{1}(t)}{t} .
\end{aligned}
$$

Taking superior limit on both sides of (32) and using (25), we have $\lim \sup _{t \rightarrow+\infty}\left(\ln x_{i}(t) / t\right) \leq g_{i}^{*}$. That is to say, if $g_{i}^{*}<0$, one can see that $\lim _{t \rightarrow+\infty} x_{i}(t)=0$ a.s. So the proof is completed.

Theorem 5. If $g_{i}^{*}=0$ and $\min _{1 \leq j \leq n} \inf _{t \in \bar{R}_{+}}\left\{a_{i j}(t)-b_{i j}\left(\Delta_{i j}^{-1}(t)\right) /\right.$ $\left.\left(1-\dot{\tau}_{i j}\left(\Delta_{i j}^{-1}(t)\right)\right)-c_{i j}^{u}\right\}>0$, then the ith population $x_{i}(t)$ of model (2) is nonpersistent in the mean a.s.

Proof. Due to (25), for any given $\varepsilon>0$, there is a positive constant $T$, such that

$$
\begin{aligned}
& t^{-1} \sum_{0<t_{k}<t} \ln \left(1+h_{i k}\right) \\
& +t^{-1} \sum_{j=1}^{n} \int_{-\tau_{i j}(0)}^{0}\left[\frac{b_{i j}\left(\Delta_{i j}^{-1}(s)\right) x_{j}(s)}{1-\dot{\tau}_{i j}\left(\Delta_{i j}^{-1}(s)\right)}\right] d s \\
& +t^{-1} \sum_{j=1}^{n} \frac{1}{r} c_{i j}^{u}\left\|\xi_{j}\right\|_{\mathscr{C}_{r}} \mu_{i j r}\left(1-e^{-r t}\right)+\frac{2 \ln k}{t} \\
& +\frac{M_{i}^{1}(t)}{t}<\varepsilon,
\end{aligned}
$$

for sufficiently large $t$ satisfying $t>T$. Substituting the above inequality into (31), therefore,

$$
\begin{aligned}
& \frac{\ln x_{i}(t)-\ln x_{i}(0)}{t}<\varepsilon \\
& -t^{-1} \int_{0}^{t} \sum_{j=1}^{n}\left(a_{i j}(s)-\frac{b_{i j}\left(\Delta_{i j}^{-1}(s)\right)}{1-\dot{\tau}_{i j}\left(\Delta_{i j}^{-1}(s)\right)}-c_{i j}^{u}\right) \\
& \cdot x_{j}(s) d s,
\end{aligned}
$$

for all $T \leq k-1 \leq t, k \geq k_{0}$ a.s.

Define $h_{i}(t)=\int_{0}^{t} x_{i}(s) d s, N=\min _{1 \leq j \leq n} \inf _{s \in \bar{R}_{+}}\left[a_{i j}(s)-\right.$ $\left.b_{i j}\left(\Delta_{i j}^{-1}(s)\right) /\left(1-\dot{\tau}_{i j}\left(\Delta_{i j}^{-1}(s)\right)\right)-c_{i j}^{u}\right]$, with the fact that $x_{i}(t) \leq$ $\sum_{i=1}^{n} x_{i}(t) \leq n|x(t)|$; we have

$$
\ln \left(\frac{d h_{i}(t)}{d t}\right)<\varepsilon t-N h_{i}(t)+\ln x_{i}(0) ; \quad t>T .
$$


Consequently,

$$
e^{N h_{i}(t)}\left(\frac{d h_{i}(t)}{d t}\right)<x_{i}(0) e^{\varepsilon t} ; \quad t>T .
$$

Integrating this inequality from $T$ to $t$,

$$
N^{-1}\left[e^{N h_{i}(t)}-e^{N h_{i}(T)}\right]<x_{i}(0) \varepsilon^{-1}\left[e^{\varepsilon t}-e^{\varepsilon T}\right] .
$$

Rewriting this inequality,

$$
e^{N h_{i}(t)}<e^{N h_{i}(T)}+x_{i}(0) N \varepsilon^{-1} e^{\varepsilon t}-x_{i}(0) N \varepsilon^{-1} e^{\varepsilon T} .
$$

Taking the logarithm of both sides, we have

$$
\begin{aligned}
& h_{i}(t) \\
& <N^{-1} \ln \left(x_{i}(0) N \varepsilon^{-1} e^{\varepsilon t}+e^{N h_{i}(T)}-x_{i}(0) N \varepsilon^{-1} e^{\varepsilon T}\right) .
\end{aligned}
$$

In other words, we can get that

$$
\begin{aligned}
& \left\{t^{-1} \int_{0}^{t} x_{i}(s) d s\right\} \leq\left\{t^{-1} N^{-1}\right. \\
& \left.\quad \cdot \ln \left(x_{i}(0) N \varepsilon^{-1} e^{\varepsilon t}+e^{N h_{i}(T)}-x_{i}(0) N \varepsilon^{-1} e^{\varepsilon T}\right)\right\}^{*} .
\end{aligned}
$$

By applying L'Hospital's rule, one can obtain

$$
\left\langle x_{i}(t)\right\rangle^{*} \leq N^{-1}\left\{t^{-1} \ln \left[x_{i}(0) N \varepsilon^{-1} e^{\varepsilon t}\right]\right\}^{*}=\frac{\varepsilon}{N} .
$$

Since $\varepsilon$ is arbitrary, we have $\left\langle x_{i}(t)\right\rangle^{*}=0$, which is the required assertion. So the proof is completed.

Theorem 6. If $g_{i}^{*}>0, \min _{1 \leq j \leq n}$ inf $_{t \in \bar{R}_{+}}\left\{a_{i j}(t)-\right.$ $\left.e^{\tau_{i j}\left(\Delta_{i j}^{-1}(t)\right)} b_{i j}\left(\Delta_{i j}^{-1}(t)\right) /\left(1-\dot{\tau}_{i j}\left(\Delta_{i j}^{-1}(t)\right)\right)-c_{i j}^{u} \mu_{i j r}\right\}>0$, then the ith population $x_{i}(t)$ of model (2) is weak persistence a.s.

Proof. To begin with, let us claim that

$$
\limsup _{t \rightarrow+\infty}\left[t^{-1} \ln x_{i}(t)\right] \leq 0 \quad \text { a.s. }
$$

Applying Itô's formula to (7), we have

$$
\begin{aligned}
& d\left(\sum_{j=1}^{n} \int_{t-\tau_{i j}(t)}^{t} \frac{e^{s+\tau_{i j}\left(\Delta_{i j}^{-1}(s)\right)} b_{i j}\left(\Delta_{i j}^{-1}(s)\right) \prod_{0<t_{k}<s}\left(1+h_{j k}\right) y_{j}(s)}{1-\dot{\tau}_{i j}\left(\Delta_{i j}^{-1}(s)\right)} d s\right. \\
& \left.+e^{t} \ln y_{i}(t)\right)=e^{t}\left[\ln x_{i}(t)-\sum_{0<t_{k}<t} \ln \left(1+h_{i k}\right)+r_{i}(t)\right. \\
& \quad-\frac{\sigma_{i}^{2}(t)}{2}-\sum_{j=1}^{n}\left(a_{i j}(t)-\frac{e^{\tau_{i j}\left(\Delta_{i j}^{-1}(t)\right)} b_{i j}\left(\Delta_{i j}^{-1}(t)\right)}{1-\dot{\tau}_{i j}\left(\Delta_{i j}^{-1}(t)\right)}\right) x_{j}(t) \\
& \left.+\sum_{j=1}^{n} c_{i j}(t) \int_{-\infty}^{0} x_{j}(t+\theta) d \mu_{i j}(\theta)-\frac{\left[\sum_{j=1}^{n} \delta_{i j}(t) x_{j}(t)\right]^{2}}{2}\right] d t \\
& +e^{t} \sigma_{i}(t) d B_{i}(t)+e^{t} \sum_{j=1}^{n} \delta_{i j}(t) x_{j}(t) d B_{i j}(t) .
\end{aligned}
$$

Thus,

$$
\begin{aligned}
& \sum_{j=1}^{n} \int_{t-\tau_{i j}(t)}^{t} \frac{e^{s+\tau_{i j}\left(\Delta_{i j}^{-1}(s)\right)} b_{i j}\left(\Delta_{i j}^{-1}(s)\right) x_{j}(s)}{1-\dot{\tau}_{i j}\left(\Delta_{i j}^{-1}(s)\right)} d s \\
& \quad-\sum_{j=1}^{n} \int_{-\tau_{i j}(0)}^{0} \frac{e^{s+\tau_{i j}\left(\Delta_{i j}^{-1}(s)\right)} b_{i j}\left(\Delta_{i j}^{-1}(s)\right) x_{j}(s)}{1-\dot{\tau}_{i j}\left(\Delta_{i j}^{-1}(s)\right)} d s+e^{t} \\
& \cdot \ln y_{i}(t)-\ln y_{i}(0)=\int_{0}^{t} e^{s}\left[\ln x_{i}(s)\right. \\
& \quad-\sum_{0<t_{k}<s} \ln \left(1+h_{i k}\right)+r_{i}(s)-\frac{\sigma_{i}^{2}(s)}{2} \\
& \quad-\sum_{j=1}^{n}\left(a_{i j}(s)-\frac{e^{\tau_{i j}\left(\Delta_{i j}^{-1}(s)\right)} b_{i j}\left(\Delta_{i j}^{-1}(s)\right)}{1-\dot{\tau}_{i j}\left(\Delta_{i j}^{-1}(s)\right)}\right) x_{j}(s) \\
& +\sum_{j=1}^{n} c_{i j}(s) \int_{-\infty}^{0} x_{j}(s+\theta) d \mu_{i j}(\theta) \\
& -\frac{\left[\sum_{j=1}^{n} \delta_{i j}(s) x_{j}(s)\right]^{2}}{2} d s+N_{i}^{1}(t)+N_{i}^{2}(t),
\end{aligned}
$$

where $N_{i}^{1}(t)=\int_{0}^{t} e^{s} \sigma_{i}(s) d B_{i}(s), \quad N_{i}^{2}(t)=$ $\int_{0}^{t} e^{s} \sum_{j=1}^{n} \delta_{i j}(s) x_{j}(s) d B_{i j}(s)$. Now we have

$$
\begin{aligned}
& \int_{0}^{t} e^{s} \sum_{j=1}^{n} c_{i j}(s) \int_{-\infty}^{0} x_{j}(s+\theta) d \mu_{i j}(\theta) d s \\
& \quad=\sum_{j=1}^{n} \int_{0}^{t} c_{i j}(s) e^{s} d s \\
& \cdot \int_{-\infty}^{-s} e^{r(s+\theta)} x_{j}(s+\theta) e^{-r(s+\theta)} d \mu_{i j}(\theta) \\
& +\sum_{j=1}^{n} \int_{-t}^{0} d \mu_{i j}(\theta) \int_{0}^{t+\theta} c_{i j}(s-\theta) e^{s-\theta} x_{j}(s) d s \\
& \leq \sum_{j=1}^{n} c_{i j}^{u} \mu_{i j r}\left\|\xi_{j}\right\|_{\mathscr{C}_{r}} t+\sum_{j=1}^{n} c_{i j}^{u} \mu_{i j r} \int_{0}^{t} e^{s} x_{j}(s) d s .
\end{aligned}
$$

Note that $N_{i}^{1}(t)(1 \leq i \leq n)$ is a local martingale with the quadratic form $\left\langle N_{i}^{1}(t), N_{i}^{1}(t)\right\rangle=\int_{0}^{t} e^{2 s} \sigma_{i}^{2}(s) d s . N_{i}^{2}(t)(1 \leq$ $i \leq n)$ is also a local martingale with the quadratic form $\left\langle N_{i}^{2}(t), N_{i}^{2}(t)\right\rangle=\int_{0}^{t} e^{2 s}\left[\sum_{j=1}^{n} \delta_{i j}(s) x_{j}(s)\right]^{2} d s$. Following from the exponential martingale inequality (26) by choosing $T_{0}=$ $\mu k, \alpha=e^{-\mu k}, \beta=\rho e^{\mu k} \ln k$,

$$
\begin{aligned}
& \mathscr{P}\left\{\sup _{0 \leq t \leq \mu k}\left[N_{i}^{\lambda}(t)-0.5 e^{-\mu k}\left\langle N_{i}^{\lambda}(t), N_{i}^{\lambda}(t)\right\rangle\right]\right. \\
& \left.>\rho e^{\mu k} \ln k\right\} \leq k^{-\rho},
\end{aligned}
$$


where $\rho>1$ and $\mu>1, \lambda=1,2$. In view of the Borel-Cantelli lemma [30], for almost all $\omega \in \Omega$, there exists a $k_{0}(\omega)$ such that, for every $k \geq k_{0}(\omega)$,

$$
\begin{aligned}
N_{i}^{\lambda}(t) \leq 0.5 e^{-\mu k}\left\langle N_{i}^{\lambda}(t), N_{i}^{\lambda}(t)\right\rangle+\rho e^{\mu k} & \ln k, \\
0 & \leq t \leq \mu k .
\end{aligned}
$$

By hypotheses (H2) and (H3), it is easy to see that there exists a constant $C$ independent of $k$ such that

$$
\begin{aligned}
& \ln x_{i}(s)-\sum_{0<t_{k}<s} \ln \left(1+h_{i k}\right)+r_{i}(s)-\frac{\sigma_{i}^{2}(s)}{2} \\
& -\sum_{j=1}^{n}\left(a_{i j}(s)-\frac{e^{\tau_{i j}\left(\Delta_{i j}^{-1}(s)\right)} b_{i j}\left(\Delta_{i j}^{-1}(s)\right)}{1-\dot{\tau}_{i j}\left(\Delta_{i j}^{-1}(s)\right)}-c_{i j}^{u} \mu_{i j r}\right) \\
& \cdot x_{j}(s)+\frac{e^{s-\mu k} \sigma_{i}^{2}(s)}{2} \\
& -\frac{\left[\sum_{j=1}^{n} \delta_{i j}(s) x_{j}(s)\right]^{2}\left[1-e^{s-\mu k}\right]}{2} \leq C,
\end{aligned}
$$

for any $0 \leq s \leq \mu k$ and $x_{i}(s)>0$. Substituting the above inequalities (45), (47), and (48) into (44), for any $0 \leq t \leq \mu k$, we have

$$
\begin{aligned}
& \sum_{j=1}^{n} \int_{t-\tau_{i j}(t)}^{t} \frac{e^{s+\tau_{i j}\left(\Delta_{i j}^{-1}(s)\right)} b_{i j}\left(\Delta_{i j}^{-1}(s)\right) x_{j}(s)}{1-\dot{\tau}_{i j}\left(\Delta_{i j}^{-1}(s)\right)} d s \\
& \quad-\sum_{j=1}^{n} \int_{-\tau_{i j}(0)}^{0} \frac{e^{s+\tau_{i j}\left(\Delta_{i j}^{-1}(s)\right)} b_{i j}\left(\Delta_{i j}^{-1}(s)\right) x_{j}(s)}{1-\dot{\tau}_{i j}\left(\Delta_{i j}^{-1}(s)\right)} d s \\
& \quad+e^{t} \ln y_{i}(t)-\ln y_{i}(0) \\
& \leq C\left[e^{t}-1\right]+\sum_{j=1}^{n} c_{i j}^{u} \mu_{i j r}\left\|\xi_{j}\right\|_{\mathscr{C}_{r}} t+2 \rho e^{\mu k} \ln k .
\end{aligned}
$$

On the other hand, we can obtain that

$$
\begin{aligned}
& e^{t} \sum_{0<t_{k}<t} \ln \left(1+h_{i k}\right)+e^{t} \ln y_{i}(t)-\ln y_{i}(0) \\
& \leq \sum_{j=1}^{n} \int_{-\tau_{i j}(0)}^{0} \frac{e^{s+\tau_{i j}\left(\Delta_{i j}^{-1}(s)\right)} b_{i j}\left(\Delta_{i j}^{-1}(s)\right) x_{j}(s)}{1-\dot{\tau}_{i j}\left(\Delta_{i j}^{-1}(s)\right)} d s \\
& \quad+e^{t} \sum_{0<t_{k}<t} \ln \left(1+h_{i k}\right)+C\left[e^{t}-1\right] \\
& \quad+\sum_{j=1}^{n} c_{i j}^{u} \mu_{i j r}\left\|\xi_{j}\right\|_{\mathscr{C}_{r}} t+2 \rho e^{\mu k} \ln k .
\end{aligned}
$$

That is to say,

$$
\begin{aligned}
& e^{t} \ln x_{i}(t)-\ln x_{i}(0) \\
& \leq \sum_{j=1}^{n} \int_{-\tau_{i j}(0)}^{0} \frac{e^{s+\tau_{i j}\left(\Delta_{i j}^{-1}(s)\right)} b_{i j}\left(\Delta_{i j}^{-1}(s)\right) x_{j}(s)}{1-\dot{\tau}_{i j}\left(\Delta_{i j}^{-1}(s)\right)} d s \\
& \quad+e^{t} \sum_{0<t_{k}<t} \ln \left(1+h_{i k}\right)+C\left[e^{t}-1\right] \\
& \quad+\sum_{j=1}^{n} c_{i j}^{u} \mu_{i j r}\left\|\xi_{j}\right\|_{\mathscr{C}_{r}} t+2 \rho e^{\mu k} \ln k .
\end{aligned}
$$

Therefore,

$$
\begin{aligned}
& \ln x_{i}(t) \\
& \leq e^{-t} \ln x_{i}(0)+\sum_{0<t_{k}<t} \ln \left(1+h_{i k}\right)+C\left[1-e^{-t}\right] \\
& \quad+2 \rho e^{\mu k-t} \ln k+e^{-t} \sum_{j=1}^{n} c_{i j}^{u} \mu_{i j r}\left\|\xi_{j}\right\|_{\mathscr{C}_{r}} t \\
& \quad+\sum_{j=1}^{n} \int_{-\tau_{i j}(0)}^{0} \frac{e^{s-t+\tau_{i j}\left(\Delta_{i j}^{-1}(s)\right)} b_{i j}\left(\Delta_{i j}^{-1}(s)\right) x_{j}(s)}{1-\dot{\tau}_{i j}\left(\Delta_{i j}^{-1}(s)\right)} d s .
\end{aligned}
$$

Consequently, if $\mu(k-1) \leq t \leq \mu k$ and $k \geq k_{0}(\omega)$, one can observe that

$$
\begin{aligned}
& t^{-1} \ln x_{i}(t) \\
& \leq t^{-1} e^{-t} \ln x_{i}(0)+t^{-1}\left(\sum_{0<t_{k}<t} \ln \left(1+h_{i k}\right)\right) \\
& \quad+t^{-1} C\left[1-e^{-t}\right]+2 t^{-1} \rho e^{\mu k-t} \ln k \\
& \quad+t^{-1} e^{-t} \sum_{j=1}^{n} c_{i j}^{u} \mu_{i j r}\left\|\xi_{j}\right\|_{\mathscr{C}_{r}} t \\
& \quad+t^{-1} \sum_{j=1}^{n} \int_{-\tau_{i j}(0)}^{0} \frac{e^{s-t+\tau_{i j}\left(\Delta_{i j}^{-1}(s)\right)} b_{i j}\left(\Delta_{i j}^{-1}(s)\right) x_{j}(s)}{1-\dot{\tau}_{i j}\left(\Delta_{i j}^{-1}(s)\right)} d s,
\end{aligned}
$$

which becomes the desired assertion (42) by letting $t \rightarrow+\infty$.

Now suppose that $g_{i}^{*}>0$; we will prove $\lim \sup _{t \rightarrow+\infty} x_{i}(t)>0$ a.s. If this assertion is not true, let $F=\left\{\limsup _{t \rightarrow+\infty} x_{i}(t)=0\right\}$ and suppose $P(F)>0$. In the light of (23), we can get that

$$
\begin{aligned}
& t^{-1}\left[\sum_{j=1}^{n} \int_{t-\tau_{i j}(t)}^{t} \frac{b_{i j}\left(\Delta_{i j}^{-1}(s)\right) x_{j}(s)}{1-\dot{\tau}_{i j}\left(\Delta_{i j}^{-1}(s)\right)} d s\right] \\
& -t^{-1}\left[\sum_{j=1}^{n} \int_{-\tau_{i j}(0)}^{0} \frac{b_{i j}\left(\Delta_{i j}^{-1}(s)\right) x_{j}(s)}{1-\dot{\tau}_{i j}\left(\Delta_{i j}^{-1}(s)\right)} d s\right]+t^{-1} \\
& \cdot \ln x_{i}(t)-t^{-1} \ln x_{i}(0)=t^{-1}\left(\sum_{0<t_{k}<t} \ln \left(1+h_{i k}\right)\right)
\end{aligned}
$$




$$
\begin{aligned}
& +t^{-1} \int_{0}^{t}\left[r_{i}(s)-\frac{\sigma_{i}^{2}(s)}{2}\right. \\
& -\sum_{j=1}^{n}\left(a_{i j}(s)-\frac{b_{i j}\left(\Delta_{i j}^{-1}(s)\right)}{1-\dot{\tau}_{i j}\left(\Delta_{i j}^{-1}(s)\right)}\right) x_{j}(s) \\
& +\sum_{j=1}^{n} c_{i j}(s) \int_{-\infty}^{0} x_{j}(s+\theta) d \mu_{i j}(\theta) \\
& \left.-\frac{\left[\sum_{j=1}^{n} \delta_{i j}(s) x_{j}(s)\right]^{2}}{2}\right] d s+\frac{M_{i}^{1}(t)}{t} \\
& +\frac{M_{i}^{2}(t)}{t} .
\end{aligned}
$$

On the other hand, for $\forall \omega \in F$, we have $\lim _{t \rightarrow+\infty} x_{i}(t, \omega)=$ 0 and the fact that $x_{i}(t) \leq \sum_{i=1}^{n} x_{i}(t) \leq n|x(t)|$. The law of large numbers for local martingales [30] indicates that $\lim _{t \rightarrow+\infty}\left(M_{i}^{2}(t) / t\right)=0$. Substituting this equality and (25) into (68),

$$
\begin{aligned}
& \limsup _{t \rightarrow+\infty}\left[t^{-1} \ln x_{i}(t, \omega)\right] \\
& \geq \limsup _{t \rightarrow+\infty} t^{-1}\left[\sum_{0<t_{k}<t} \ln \left(1+h_{i k}\right)\right. \\
& \left.+\int_{0}^{t}\left(r_{i}(s)-\frac{\sigma_{i}^{2}(s)}{2}\right) d s\right]=g_{i}^{*}>0 \text {. }
\end{aligned}
$$

Then $\mathscr{P}\left(\lim \sup _{t \rightarrow+\infty}\left[t^{-1} \ln x_{i}(t)\right]>0\right)>0$, which contradicts (42). So the proof is completed.

When it comes to the study of population model, the role of stochastic permanence indicating the eternal existence of the population can never be ignorant with its theoretical and practical significance. So now let us show that the population $x_{i}(t)$ is stochastic permanence in some cases.

(H5): there are two positive constants $m$ and $M$ such that $m \leq \prod_{0<t_{k}<t}\left(1+h_{i k}\right) \leq M(i=1,2, \ldots, n)$ for all $t>0$.

Theorem 7. Under assumption (H5), if $\widetilde{r}_{i}^{l} \geq 2\left(\bar{\sigma}_{i}^{u}\right)^{2}, r \geq 1$, and there exists $\varepsilon_{2} \in(0,2 r)$ such that $\min _{1 \leq i, j \leq n} \inf _{t \in \bar{R}_{+}}\left\{e^{\varepsilon_{2}\left(t+\tau_{i j}(t)\right)}-e^{\varepsilon_{2}\left(\Delta_{i j}^{-1}(t)\right)} /\left(1-\dot{\tau}_{i j}(t)\right)\right\} \geq 0$, then the population $x(t)$ of model (2) is stochastic permanence.

Proof. We prove that, for arbitrary $\varepsilon \in(0,1)$, there is a positive constant $H=H(\varepsilon)$ such that $\liminf _{t \rightarrow+\infty} \mathscr{P}\{|x(t)| \leq$ $H\} \geq 1-\varepsilon$. Consider $0<p<1$; by Itô's formula, we have

$$
\begin{gathered}
d \sum_{i=1}^{n} y_{i}^{p}(t) \leq F(x(t)) d t-\left[\sum_{i=1}^{n} \frac{\varepsilon_{2} x_{i}^{p}(t)}{\left(\prod_{0<t_{k}<t}\left(1+h_{i k}\right)\right)^{p}}\right. \\
+\sum_{i=1}^{n} \sum_{j=1}^{n} e^{\varepsilon_{2} \tau_{i j}(t)} x_{j}^{2}(t)-\sum_{i=1}^{n} \sum_{j=1}^{n} x_{j}^{2}\left(t-\tau_{i j}(t)\right)
\end{gathered}
$$

$$
\begin{aligned}
& -\sum_{i=1}^{n} \sum_{j=1}^{n} \int_{-\infty}^{0} x_{j}^{2}(t+\theta) d \mu_{i j}(\theta) \\
& \left.+\sum_{i=1}^{n} \sum_{j=1}^{n} \mu_{i j r} x_{j}^{2}(t)\right] d t \\
& +\sum_{i=1}^{n} \frac{p x_{i}^{p}(t) \sigma_{i}(t)}{\left(\prod_{0<t_{k}<t}\left(1+h_{i k}\right)\right)^{p}} d B_{i}(t) \\
& +\sum_{i=1}^{n} \frac{p x_{i}^{p}(t)}{\left(\prod_{0<t_{k}<t}\left(1+h_{i k}\right)\right)^{p}} \\
& \cdot\left[\sum_{j=1}^{n} \delta_{i j}(t) x_{j}(t)\right] d B_{i j}(t),
\end{aligned}
$$

where

$$
\begin{aligned}
F(x(t))= & \sum_{i=1}^{n} \sum_{j=1}^{n} e^{\varepsilon_{2} \tau_{i j}(t)} x_{j}^{2}(t) \\
& +\sum_{i=1}^{n} \frac{\left(\varepsilon_{2}+r_{i}(t) p\right) x_{i}^{p}(t)}{\left(\prod_{0<t_{k}<t}\left(1+h_{i k}\right)\right)^{p}} \\
& +\sum_{i=1}^{n} \sum_{j=1}^{n} \frac{p^{2} b_{i j}^{2}(t) x_{i}^{2 p}(t)}{4\left(\prod_{0<t_{k}<t}\left(1+h_{i k}\right)\right)^{2 p}} \\
& +\sum_{i=1}^{n} \sum_{j=1}^{n} \mu_{i j r} x_{j}^{2}(t)+\sum_{i=1}^{n} \sum_{j=1}^{n} x_{j}^{2}\left(t-\tau_{i j}(t)\right) \\
& +\sum_{i=1}^{n} \sum_{j=1}^{n} \frac{p^{2} c_{i j}^{2}(t) x_{i}^{2 p}(t)}{4\left(\prod_{0<t_{k}<t}\left(1+h_{i k}\right)\right)^{2 p}} \\
& -\sum_{i=1}^{n} \frac{1}{2} p(1-p) \frac{\sigma_{i}^{2}(t) x_{i}^{p}(t)}{\left(\prod_{0<t_{k}<t}\left(1+h_{i k}\right)\right)^{p}} \\
& -\sum_{i=1}^{n} \frac{1}{2} p(1-p) \frac{x_{i}^{p}(t)}{\left(\prod_{0<t_{k}<t}\left(1+h_{i k}\right)\right)^{p}} \\
& \left.\cdot \sum_{j=1}^{n} \delta_{i j}(t) x_{j}(t)\right]^{2}
\end{aligned}
$$

With the fact that $x_{i}(t) \leq \sum_{i=1}^{n} x_{i}(t) \leq n|x(t)|$, it is easy to see that $F(x(t))$ is bounded in $R_{+}^{n}$; in other words, $M_{1}=$ $\sup _{x(t) \in R_{+}^{n}} F(x(t))<+\infty$. Therefore,

$$
\begin{aligned}
& d \sum_{i=1}^{n} y_{i}^{p}(t) \leq\left[M_{1}-\sum_{i=1}^{n} \frac{\varepsilon_{2} x_{i}^{p}(t)}{\left(\prod_{0<t_{k}<t}\left(1+h_{i k}\right)\right)^{p}}\right. \\
& \left.\quad-\sum_{i=1}^{n} \sum_{j=1}^{n} e^{\varepsilon_{2} \tau_{i j}(t)} x_{j}^{2}(t)+\sum_{i=1}^{n} \sum_{j=1}^{n} x_{j}^{2}\left(t-\tau_{i j}(t)\right)\right] d t
\end{aligned}
$$




$$
\begin{aligned}
& +\sum_{i=1}^{n} \sum_{j=1}^{n} \int_{-\infty}^{0} x_{j}^{2}(t+\theta) d \mu_{i j}(\theta) d t \\
& -\sum_{i=1}^{n} \sum_{j=1}^{n} \mu_{i j r} x_{j}^{2}(t) d t+p x_{i}^{p}(t) \sigma_{i}(t) d B_{i}(t) \\
& +p x_{i}^{p}(t)\left(\sum_{j=1}^{n} \delta_{i j}(t) x_{j}(t)\right) d B_{i j}(t) .
\end{aligned}
$$

Once again by Itô's formula, we have

$$
\begin{aligned}
d\left[e^{\varepsilon_{2} t} \sum_{i=1}^{n} y_{i}^{p}(t)\right]=e^{\varepsilon_{2} t}\left[\sum_{i=1}^{n} \varepsilon_{2} y_{i}^{p}(t) d t+d \sum_{i=1}^{n} y_{i}^{p}(t)\right] \\
\quad \leq e^{\varepsilon_{2} t}\left[M_{1}-\sum_{i=1}^{n} \sum_{j=1}^{n} e^{\varepsilon_{2} \tau_{i j}(t)} x_{j}^{2}(t)\right. \\
+\sum_{i=1}^{n} \sum_{j=1}^{n} x_{j}^{2}\left(t-\tau_{i j}(t)\right) \\
+\sum_{i=1}^{n} \sum_{j=1}^{n} \int_{-\infty}^{0} x_{j}^{2}(t+\theta) d \mu_{i j}(\theta) \\
\left.\quad-\sum_{i=1}^{n} \sum_{j=1}^{n} \mu_{i j r} x_{j}^{2}(t)\right] d t+e^{\varepsilon_{2} t} p x_{i}^{p}(t) \sigma_{i}(t) d B_{i}(t) \\
+e^{\varepsilon_{2} t} p x_{i}^{p}(t)\left(\sum_{j=1}^{n} \delta_{i j}(t) x_{j}(t)\right) d B_{i j}(t) .
\end{aligned}
$$

We hence derive that

$$
\begin{aligned}
e^{\varepsilon_{2} t} E & {\left[\sum_{i=1}^{n} y_{i}^{p}(t)\right] } \\
\leq & \sum_{i=1}^{n} \xi_{i}^{p}(0)+\frac{e^{\varepsilon_{2} t} M_{1}}{\varepsilon_{2}}-\frac{M_{1}}{\varepsilon_{2}} \\
& -E \sum_{i=1}^{n} \sum_{j=1}^{n} \int_{0}^{t} e^{\varepsilon_{2} s+\varepsilon_{2} \tau_{i j}(s)} x_{j}^{2}(s) d s \\
& +E \sum_{i=1}^{n} \sum_{j=1}^{n} \int_{-\tau_{i j}(0)}^{t-\tau_{i j}(t)} \frac{e^{\varepsilon_{2} \Delta_{i j}^{-1}(s)} x_{j}^{2}(s) d s}{1-\dot{\tau}_{i j}(s)} d s \\
& +E \sum_{i=1}^{n} \sum_{j=1}^{n} \int_{0}^{t} e^{\varepsilon_{2} s} \int_{-\infty}^{0} x_{j}^{2}(s+\theta) d \mu_{i j}(\theta) d s \\
& -E \sum_{i=1}^{n} \sum_{j=1}^{n} \int_{0}^{t} \mu_{i j r} e^{\varepsilon_{2} s} x_{j}^{2}(s) d s
\end{aligned}
$$

$$
\begin{aligned}
& \leq \sum_{i=1}^{n} \xi_{i}^{p}(0)+\frac{e^{\varepsilon_{2} t} M_{1}}{\varepsilon_{2}}-\frac{M_{1}}{\varepsilon_{2}} \\
& \quad+E \sum_{i=1}^{n} \sum_{j=1}^{n} \int_{-\tau_{i j}(0)}^{0} e^{\varepsilon_{2} s+\varepsilon_{2} \tau_{i j}(s)} x_{j}^{2}(s) d s \\
& \quad+E \sum_{i=1}^{n} \sum_{j=1}^{n} \int_{0}^{t} e^{\varepsilon_{2} s} \int_{-\infty}^{0} x_{j}^{2}(s+\theta) d \mu_{i j}(\theta) d s \\
& \quad-E \sum_{i=1}^{n} \sum_{j=1}^{n} \mu_{i j r} \int_{0}^{t} e^{\varepsilon_{2} s} x_{j}^{2}(s) d s .
\end{aligned}
$$

From hypothesis (H1), we have

$$
\begin{aligned}
& \int_{0}^{t} e^{\varepsilon_{2} s} \int_{-\infty}^{0} x_{j}^{2}(s+\theta) d \mu_{i j}(\theta) d s \\
& =\int_{0}^{t} e^{\varepsilon_{2} s} d s \int_{-\infty}^{-s} e^{2 r(s+\theta)} x_{j}^{2}(s+\theta) e^{-2 r(s+\theta)} d \mu_{i j}(\theta) \\
& \quad+\int_{-t}^{0} d \mu_{i j}(\theta) \int_{0}^{t+\theta} e^{\varepsilon_{2}(s-\theta)} x_{j}^{2}(s) d s \\
& \leq\left\|\xi_{j}\right\|_{\mathscr{C}_{r}}^{2} \mu_{i j r} t+\mu_{i j r} \int_{0}^{t} e^{\varepsilon_{2} s} x_{j}^{2}(s) d s
\end{aligned}
$$

This implies that $\lim \sup _{t \rightarrow+\infty} E\left[\sum_{i=1}^{n} y_{i}^{p}(t)\right] \leq M_{1} / \varepsilon_{2}$. Therefore,

$$
\limsup _{t \rightarrow+\infty} E\left[\sum_{i=1}^{n}\left(\prod_{0<t_{k}<t}\left(1+h_{i k}\right)\right)^{p} y_{i}^{p}(t)\right] \leq M^{p} \frac{M_{1}}{\varepsilon_{2}} .
$$

So we can get that

$$
\limsup _{t \rightarrow+\infty} E\left[\sum_{i=1}^{n} x_{i}^{p}(t)\right] \leq M^{p} \frac{M_{1}}{\varepsilon_{2}} .
$$

On the other hand, we have $|x(t)|^{2} \leq n \max _{1 \leq i \leq n} \sup _{t \in R} x_{i}(t)^{2}$, so

$$
|x(t)|^{p} \leq n^{p / 2} \max _{1 \leq i \leq n} \sup _{t \in R} x_{i}(t)^{p} \leq n^{p / 2} \sum_{i=1}^{n} x_{i}^{p}(t) .
$$

Therefore, finally we have

$$
\limsup _{t \rightarrow+\infty} E|x(t)|^{p} \leq n^{p / 2} M^{p} \frac{M_{1}}{\varepsilon_{2}} .
$$

Setting $K=n^{p / 2} M^{p}\left(M_{1} / \varepsilon_{2}\right)$ and choosing $p=1 / 2$,

$$
\limsup _{t \rightarrow+\infty} E(\sqrt{|x(t)|}) \leq K \text {. }
$$

Now for any $\varepsilon>0$, let $H=K^{2} / \varepsilon^{2}$. Then, by Chebyshev's inequality,

$$
\begin{aligned}
\mathscr{P}\{|x(t)|>H\} & =\mathscr{P}\{\sqrt{|x(t)|}>\sqrt{H}\} \\
& \leq \frac{E(\sqrt{|x(t)|})}{\sqrt{H}} .
\end{aligned}
$$


Hence lim $\sup _{t \rightarrow+\infty} \mathscr{P}\{|x(t)|>H\} \leq \varepsilon$. This implies

$$
\liminf _{t \rightarrow+\infty} \mathscr{P}\{|x(t)| \leq H\} \geq 1-\varepsilon .
$$

Next, we claim that, for arbitrary $\varepsilon>0$, there is a constant $\beta>0$ such that $\liminf _{t \rightarrow+\infty} \mathscr{P}\{|x(t)| \geq \beta\} \geq 1-\varepsilon$. Denote $V(y(t))=\sum_{i=1}^{n} y_{i}(t)$. Applying Itôs formula, we get

$$
\begin{aligned}
& d V(y(t))=\sum_{i=1}^{n} y_{i}(t)\left[r_{i}(t)-\sum_{j=1}^{n} a_{i j}(t)\right. \\
& \cdot \prod_{0<t_{k}<t}\left(1+h_{j k}\right) y_{j}(t)+\sum_{j=1}^{n} b_{i j}(t) \\
& \cdot \prod_{0<t_{k}<t-\tau_{i j}(t)}\left(1+h_{j k}\right) y_{j}\left(t-\tau_{i j}(t)\right)+\sum_{j=1}^{n} c_{i j}(t) \\
& \left.\cdot \int_{-\infty}^{0} \prod_{0<t_{k}<t+\theta}\left(1+h_{j k}\right) y_{j}(t+\theta) d \mu_{i j}(\theta)\right] d t \\
& +\sum_{i=1}^{n} y_{i}(t) \sigma_{i}(t) d B_{i}(t)+\sum_{i=1}^{n} y_{i}(t) \sum_{j=1}^{n} \delta_{i j}(t) \\
& \cdot \prod_{0<t_{k}<t}\left(1+h_{j k}\right) y_{j}(t) d B_{i j}(t) .
\end{aligned}
$$

Define $U(y(t))=1 / V(y(t))$ on $t \geq 0$. Then we have

$$
\begin{aligned}
& d U=\left[-U^{2}\left(\sum _ { i = 1 } ^ { n } y _ { i } ( t ) \left[r_{i}(t)-\sum_{j=1}^{n} a_{i j}(t)\right.\right.\right. \\
& \cdot \prod_{0<t_{k}<t}\left(1+h_{j k}\right) y_{j}(t)+\sum_{j=1}^{n} b_{i j}(t) \\
& \cdot \prod_{0<t_{k}<t-\tau_{i j}(t)}\left(1+h_{j k}\right) y_{j}\left(t-\tau_{i j}(t)\right)+\sum_{j=1}^{n} c_{i j}(t) \\
& \left.\left.\cdot \int_{-\infty}^{0} \prod_{0<t_{k}<t+\theta}\left(1+h_{j k}\right) y_{j}(t+\theta) d \mu_{i j}(\theta)\right]\right) \\
& +U^{3}\left(\left[\sum_{i=1}^{n} y_{i}(t) \sigma_{i}(t)\right]^{2}+\left[\sum_{i=1}^{n} y_{i}(t) \sum_{j=1}^{n} \delta_{i j}(t)\right.\right. \\
& \left.\left.\left.\cdot \prod_{0<t_{k}<t}\left(1+h_{j k}\right) y_{j}(t)\right]^{2}\right)\right] d t-U^{2} \sum_{i=1}^{n} y_{i}(t) \\
& \cdot \sigma_{i}(t) d B_{i}(t)-U^{2} \sum_{i=1}^{n} y_{i}(t) \sum_{j=1}^{n} \delta_{i j}(t) \\
& \cdot \prod_{0<t_{k}<t}\left(1+h_{j k}\right) y_{j}(t) d B_{i j}(t),
\end{aligned}
$$

dropping $y(t)$ from $U(y(t))$. Define the function $\bar{V}(y(t))=$ $U^{2+p}(y(t))$; then by Itô's formula, we have

$$
\begin{aligned}
& L \bar{V}(y(t))=(2+p) U^{p}\left[-U^{3}\left(\sum _ { i = 1 } ^ { n } y _ { i } ( t ) \left[r_{i}(t)\right.\right.\right. \\
& -\sum_{j=1}^{n} a_{i j}(t) \prod_{0<t_{k}<t}\left(1+h_{j k}\right) y_{j}(t)+\sum_{j=1}^{n} b_{i j}(t) \\
& \cdot \prod_{0<t_{k}<t-\tau_{i j}(t)}\left(1+h_{j k}\right) y_{j}\left(t-\tau_{i j}(t)\right)+\sum_{j=1}^{n} c_{i j}(t) \\
& \left.\left.\cdot \int_{-\infty}^{0} \prod_{0<t_{k}<t+\theta}\left(1+h_{j k}\right) y_{j}(t+\theta) d \mu_{i j}(\theta)\right]\right) \\
& +U^{4}\left(\left[\sum_{i=1}^{n} y_{i}(t) \sigma_{i}(t)\right]^{2}+\left[\sum_{i=1}^{n} y_{i}(t) \sum_{j=1}^{n} \delta_{i j}(t)\right.\right. \\
& \left.\left.\cdot \prod_{0<t_{k}<t}\left(1+h_{j k}\right) y_{j}(t)\right]^{2}\right)+\frac{p+1}{2} \\
& \cdot U^{4}\left(\left[\sum_{i=1}^{n} y_{i}(t) \sigma_{i}(t)\right]^{2}+\left[\sum_{i=1}^{n} y_{i}(t) \sum_{j=1}^{n} \delta_{i j}(t)\right.\right. \\
& \left.\left.\left.\cdot \prod_{0<t_{k}<t}\left(1+h_{j k}\right) y_{j}(t)\right]^{2}\right)\right]
\end{aligned}
$$

Since we know that

$$
\begin{aligned}
& U \sum_{i=1}^{n} y_{i}(t) r_{i}(t) \geq \vec{r}_{i}^{l}, \\
& U^{2} \sum_{i=1}^{n} y_{i}(t) \sum_{j=1}^{n} a_{i j}(t) \prod_{0<t_{k}<t}\left(1+h_{j k}\right) y_{j}(t) \leq M \bar{a}_{i j}^{u}, \\
& U^{2}\left(\sum_{i=1}^{n} y_{i}(t) \sigma_{i}(t)\right)^{2} \leq\left(\bar{\sigma}_{i}^{u}\right)^{2} \\
& U^{4}\left(\sum_{i=1}^{n} y_{i}(t) \sum_{j=1}^{n} \delta_{i j}(t) \prod_{0<t_{k}<t}\left(1+h_{j k}\right) y_{j}(t)\right)^{2} \\
& \quad \leq M^{2}\left(\bar{\delta}_{i j}^{u}\right)^{2} .
\end{aligned}
$$

It follows from (71) that

$$
\begin{aligned}
& L \bar{V}(y(t)) \leq(2+p)\left(-\left(\vec{r}_{i}^{l}-\frac{p+3}{2}\left(\bar{\sigma}_{i}^{u}\right)^{2}\right) U^{2+p}\right. \\
& \left.+M \bar{a}_{i j}^{u} U^{1+p}+\frac{p+3}{2} M^{2}\left(\bar{\delta}_{i j}^{u}\right)^{2} U^{p}\right) .
\end{aligned}
$$


Now choose a constant $k>0$ sufficiently small such that it satisfies $k-(2+p)\left(\vec{r}_{i}^{l}-((p+3) / 2)\left(\bar{\sigma}_{i}^{u}\right)^{2}\right)<0$. Therefore,

$$
\begin{aligned}
& L\left[e^{k t} \bar{V}(y(t))\right]=k e^{k t} \bar{V}(y(t))+e^{k t} L \bar{V}(y(t)) \\
& \quad \leq k e^{k t} U^{2+p}(y(t))+e^{k t}(2+p) \\
& \quad \cdot\left(-\left(\bar{r}_{i}^{l}-\frac{p+3}{2}\left(\bar{\sigma}_{i}^{u}\right)^{2}\right) U^{2+p}\right. \\
& \left.+M \bar{a}_{i j}^{u} U^{1+p}+\frac{p+3}{2} M^{2}\left(\bar{\delta}_{i j}^{u}\right)^{2} U^{p}\right) \\
& \quad=e^{k t}\left(\left[k-(2+p)\left(\bar{r}_{i}^{l}-\frac{p+3}{2}\left(\bar{\sigma}_{i}^{u}\right)^{2}\right)\right] U^{2+p}\right. \\
& +(2+p) M \bar{a}_{i j}^{u} U^{1+p} \\
& \left.+(2+p) \frac{p+3}{2} M^{2}\left(\bar{\delta}_{i j}^{u}\right)^{2} U^{p}\right) \leq K e^{k t} .
\end{aligned}
$$

This implies that

$$
\limsup _{t \rightarrow+\infty} E U^{2+p}(y(t)) \leq K
$$

For $y(t) \in R_{+}^{n}$, note that $\left(\sum_{i=1}^{n} y_{i}(t)\right)^{2+p} \leq n^{2+p}|y(t)|^{2+p}$. Consequently

$$
\begin{aligned}
& \limsup _{t \rightarrow+\infty} E \frac{1}{|x(t)|^{2+p}} \leq n^{-2-p} \limsup _{t \rightarrow+\infty} E \frac{1}{\left(\sum_{i=1}^{n} x_{i}(t)\right)^{2+p}} \\
& \quad=n^{-2-p} \limsup _{t \rightarrow+\infty} E \frac{1}{\left(\sum_{i=1}^{n} \prod_{0<t_{k}<t}\left(1+h_{i k}\right) y_{i}(t)\right)^{2+p}} \\
& \quad \leq(m n)^{-2-p} K=: d .
\end{aligned}
$$

So for any $\varepsilon>0$, setting $\beta=(\varepsilon / d)^{1 /(2+p)}$, by Chebyshev's inequality, gets that

$$
\begin{aligned}
\mathscr{P}\{|x(t)|<\beta\} & =\mathscr{P}\left\{|x(t)|^{2+p}<\beta^{2+p}\right\} \\
& =\mathscr{P}\left\{\frac{1}{|x(t)|^{2+p}}>\frac{1}{\beta^{2+p}}\right\} \\
& \leq \frac{E\left[1 /|x(t)|^{2+p}\right]}{1 / \beta^{2+p}} \\
& =\beta^{2+p} E\left[\frac{1}{|x(t)|^{2+p}}\right],
\end{aligned}
$$

which means that $\limsup _{t \rightarrow+\infty} \mathscr{P}\{|x(t)|<\beta\} \leq \beta^{2+p} d=\varepsilon$. We can get that

$$
\liminf _{t \rightarrow+\infty} \mathscr{P}\{|x(t)| \geq \beta\} \geq 1-\varepsilon
$$

So the whole proof is completed.

Remark 8. If $g_{i}^{*}>0$ and $\min _{1 \leq j \leq n} \inf _{t \in \bar{R}_{+}}\left\{a_{i j}(t)-\right.$ $\left.e^{\tau_{i j}\left(\Delta_{i j}^{-1}(t)\right)} b_{i j}\left(\Delta_{i j}^{-1}(t)\right) /\left(1-\dot{\tau}_{i j}\left(\Delta_{i j}^{-1}(t)\right)\right)-c_{i j}^{u} \mu_{i j r}\right\}>0$, then the population $x_{i}(t)$ will be weak persistence. If $g_{i}^{*}<0$ and $\min _{1 \leq j \leq n} \inf _{t \in \bar{R}_{+}}\left\{a_{i j}(t)-b_{i j}\left(\Delta_{i j}^{-1}(t)\right) /\left(1-\dot{\tau}_{i j}\left(\Delta_{i j}^{-1}(t)\right)\right)-c_{i j}^{u}\right\} \geq 0$, then the population $x_{i}(t)$ will go to extinction. That is to say, if $\min _{1 \leq j \leq n} \inf _{t \in \bar{R}_{+}}\left\{a_{i j}(t)-e^{\tau_{i j}\left(\Delta_{i j}^{-1}(t)\right)} b_{i j}\left(\Delta_{i j}^{-1}(t)\right) /\left(1-\dot{\tau}_{i j}\left(\Delta_{i j}^{-1}(t)\right)\right)-\right.$ $\left.c_{i j}^{u} \mu_{i j r}\right\}>0$ and $\min _{1 \leq j \leq n} \inf _{t \in \bar{R}_{+}}\left\{a_{i j}(t)-b_{i j}\left(\Delta_{i j}^{-1}(t)\right) /(1-\right.$ $\left.\left.\dot{\tau}_{i j}\left(\Delta_{i j}^{-1}(t)\right)\right)-c_{i j}^{u}\right\} \geq 0$ hold, then $g_{i}^{*}$ is the critical number between weak persistence and extinction for the population $x_{i}(t)$.

Remark 9. Generally speaking, as the biology has implied, in Theorem 4, on one hand, if the species in the process of planting, that is, $h_{i k}>0$, or harvesting, that is, $h_{i k}<0$, is affected by stochastic environmental noises which plays a dominant role, then the species will be extinct a.s. In a word, population probably will come to an end in the worst cases which is revealed in Theorem 4, while if the growth rate and the influences of the stochastic noises and impulsive perturbations cancel each other out, then the effects of interspecific (for $i \neq j$ ) and intraspecific (for $i=j$ ) interaction at time $t$, that is, $a_{i j}(t)$, are the dominant factor. So the living chances are considerably rare which is shown in Theorem 5. In Theorem 6, even though the growth rate is larger than the influences of the stochastic noises and impulsive perturbations, $a_{i j}(t)$ plays the dominant role; then the population size is limited to zero with the time permitted; however, the opportunity of the survival of it still exists. In Theorem 7, if the growth rate is large enough, then the species will be stochastic permanence. This can well explain why the conditions are gradually stronger from Theorems 4-6.

Remark 10. According to $g_{i}^{*}=$ $\lim \sup _{t \rightarrow+\infty} t^{-1}\left[\sum_{0<t_{k}<t} \ln \left(1+h_{i k}\right)+\int_{0}^{t}\left(r_{i}(s)-\sigma_{i}^{2}(s) / 2\right) d s\right]$, on one hand, we are conscious of the fact that the stochastic noise on $r_{i}(t)$ is detrimental to the survival of the population but the stochastic noise on $a_{i j}(t)$ has hardly impressed on the persistence or extinction of the population. Thus, in true ecological modelling, the stochastic noise on $r_{i}(t)$ should be realized but the stochastic noise on $a_{i j}(t)$ could be overlooked in some cases.

Remark 11. In view of $g_{i}^{*}=\limsup _{t \rightarrow+\infty} t^{-1}\left[\sum_{0<t_{k}<t} \ln (1+\right.$ $\left.\left.h_{i k}\right)+\int_{0}^{t}\left(r_{i}(s)-\sigma_{i}^{2}(s) / 2\right) d s\right]$, we can find that the properties including extinction, nonpersistence in the mean, weak persistence, and stochastic permanence are not affected by the impulsive perturbations which are bounded and if the impulsive perturbations are unbounded the properties will change significantly.

Remark 12. Assumption (H5) is easy to be satisfied. For example, for the $i$ th population, if $h_{i k}=e^{(-1)^{k+1} / k^{2}}-1$, then $e^{0.75}<\prod_{0<t_{k}<t}\left(1+h_{i k}\right)<e$ for all $t>0$. Thus $1 \leq \prod_{0<t_{k}<t}(1+$ $\left.h_{i k}\right) \leq e$ for all $t>0$.

\section{Examples and Numerical Simulations}

In this section, we explore system behavior numerical solutions of model (2). For convenience, consider the case $n=2$ 
and let the probability measure $\mu_{i j}(\theta)=e^{\theta}(i=1,2, j=1,2)$ on $(-\infty, 0]$ satisfying $\mu_{i j r}=\int_{-\infty}^{0} e^{-2 r \theta} d \mu_{i j}(\theta)<+\infty$. Thus the nonautonomous stochastic model (2) will be written as

$$
\begin{aligned}
& d x(t)=x(t)\left[r_{1}(t)-a_{11}(t) x(t)-a_{12}(t) y(t)\right. \\
& +b_{11}(t) x\left(t-\tau_{11}(t)\right)+b_{12}(t) y\left(t-\tau_{12}(t)\right) \\
& +r e^{t} c_{11}(t) \int_{-\infty}^{0} e^{r \theta} \xi_{1}(\theta) d \theta \\
& +r e^{t} c_{12}(t) \int_{-\infty}^{0} e^{r \theta} \xi_{2}(\theta) d \theta \\
& +r e^{t} c_{11}(t) \int_{0}^{t} e^{r \theta} x(\theta) d \theta \\
& \left.+r e^{t} c_{12}(t) \int_{0}^{t} e^{r \theta} y(\theta) d \theta\right] d t+\sigma_{1}(t)(t) \\
& \cdot x(t) d B_{1}(t)+\delta_{11}(t) x^{2}(t) d B_{11}(t) \\
& +\delta_{12}(t) x(t) y(t) d B_{12}(t) \text {, } \\
& d y(t)=y(t)\left[r_{2}(t)-a_{21}(t) x(t)-a_{22}(t) y(t)\right. \\
& +b_{21}(t) x\left(t-\tau_{21}(t)\right)+b_{22}(t) y\left(t-\tau_{22}(t)\right) \\
& +r e^{t} c_{21}(t) \int_{-\infty}^{0} e^{r \theta} \xi_{1}(\theta) d \theta \\
& +r e^{t} c_{22}(t) \int_{-\infty}^{0} e^{r \theta} \xi_{2}(\theta) d \theta \\
& +r e^{t} c_{21}(t) \int_{0}^{t} e^{r \theta} x(\theta) d \theta \\
& \left.+r e^{t} c_{22}(t) \int_{0}^{t} e^{r \theta} y(\theta) d \theta\right] d t+\sigma_{2}(t)(t) \\
& \cdot y(t) d B_{2}(t)+\delta_{21}(t) x(t) y(t) d B_{21}(t) \\
& +\delta_{22}(t) y^{2}(t) d B_{22}(t), \\
& x(\theta)=\xi_{1}(\theta), \\
& y(\theta)=\xi_{2}(\theta), \\
& \xi(\theta)=\left(\xi_{1}(\theta), \xi_{2}(\theta)\right)^{T} \in \mathscr{C}_{g} .
\end{aligned}
$$

By employing the Milstein method mentioned in Higham [31] to discretize the two equations, where the integral term is approximated by using the composite $\theta$-rule as a quadrature [32] and taking $\xi_{1}(\theta)=e^{-0.5 \theta}, \xi_{2}(\theta)=2 e^{-0.5 \theta}, \tau_{i j}(t) \equiv$ $0.3(i=1,2, j=1,2)$, we can obtain the discrete approximate solution with respect to (79)

$$
\begin{aligned}
& x_{k+1}=x_{k}+x_{k}\left[r_{1}(k \Delta t)-a_{11}(k \Delta t) x_{k}\right. \\
& -a_{12}(k \Delta t) y_{k}+b_{11}(k \Delta t) x_{k-300}+b_{12}(k \Delta t) y_{k-300}
\end{aligned}
$$

$$
\begin{aligned}
& +\frac{c_{11}(k \Delta t) r e^{-k \Delta t}}{2}+c_{12}(k \Delta t) r e^{-k \Delta t} \\
& +c_{11}(k \Delta t) r e^{-k \Delta t} \sum_{j=1}^{k} e^{-r j \Delta t} x_{j} \Delta t \\
& \left.+c_{12}(k \Delta t) r e^{-k \Delta t} \sum_{j=1}^{k} e^{-r j \Delta t} x_{j} \Delta t\right] \Delta t+\sigma_{1}(k \Delta t) \\
& \cdot x_{k} \sqrt{\Delta t} \zeta_{1 k}+\frac{1}{2} \sigma_{1}^{2}(k \Delta t) x_{k}\left[\zeta_{1 k}^{2}-1\right] \Delta t \\
& +\delta_{11}(k \Delta t) x_{k}^{2} \sqrt{\Delta t} \zeta_{11 k}+\frac{1}{2} \delta_{11}^{2}(k \Delta t) x_{k}^{2}\left[\zeta_{11 k}^{2}-1\right] \\
& \cdot \Delta t+\delta_{12}(k \Delta t) x_{k} y_{k} \sqrt{\Delta t} \zeta_{12 k}+\frac{1}{2} \delta_{12}^{2}(k \Delta t) \\
& \cdot x_{k} y_{k}\left[\zeta_{12 k}^{2}-1\right] \Delta t \\
& y_{k+1}=y_{k}+y_{k}\left[r_{2}(k \Delta t)-a_{21}(k \Delta t) x_{k}\right. \\
& -a_{22}(k \Delta t) y_{k}+b_{21}(k \Delta t) x_{k-300}+b_{22}(k \Delta t) y_{k-300} \\
& +\frac{c_{21}(k \Delta t) r e^{-k \Delta t}}{2}+c_{22}(k \Delta t) r e^{-k \Delta t} \\
& +c_{21}(k \Delta t) r e^{-k \Delta t} \sum_{j=1}^{k} e^{-r j \Delta t} x_{j} \Delta t \\
& \left.+c_{22}(k \Delta t) r e^{-k \Delta t} \sum_{j=1}^{k} e^{-r j \Delta t} y_{j} \Delta t\right] \Delta t+\sigma_{2}(k \Delta t) \\
& \cdot y_{k} \sqrt{\Delta t} \zeta_{2 k}+\frac{1}{2} \sigma_{2}^{2}(k \Delta t) y_{k}\left[\zeta_{2 k}^{2}-1\right] \Delta t \\
& +\delta_{21}(k \Delta t) x_{k} y_{k} \sqrt{\Delta t} \zeta_{21 k}+\frac{1}{2} \delta_{21}^{2}(k \Delta t) x_{k} y_{k}\left[\zeta_{21 k}^{2}\right. \\
& -1] \Delta t+\delta_{22}(k \Delta t) y_{k}^{2} \sqrt{\Delta t} \zeta_{22 k}+\frac{1}{2} \delta_{22}^{2}(k \Delta t) \\
& \cdot y_{k}^{2}\left[\zeta_{22 k}^{2}-1\right] \Delta t
\end{aligned}
$$

where $\zeta_{i k}$ and $\zeta_{i j k}(i=1,2, j=1,2, k=1,2, \ldots, n)$ are the Gaussian random variables which follow $N(0,1)$.

Here, we choose $t_{k}=100 \mathrm{k}, a_{i j}(t)=1.2+0.01 \cos t(i=$ $1,2, j=1,2), b_{i j}(t)=0.3+0.05 \sin t, c_{i j}(t)=0.006+0.02 \cos t$, $\sigma_{i}(t)=1.5, \delta_{i j}(t)=0.1, h_{i k}=e^{-0.005}-1$ for all $k \in N$ and step size $\Delta t=0.001$. The only difference between conditions of Figures $1(\mathrm{a})-1(\mathrm{~d})$ is that the representations of $r_{i}(t)$ are different.

Because of $-1<h_{i k}=e^{-0.005}-1<0$, both species $x(t)$ and $y(t)$ are in the process of harvesting. Moreover, in Figure 1(a), we choose $r_{i}(t)=0.1+0.4 \sin t$; stochastic environmental noises play a dominant role; then the conditions of Theorem 4 


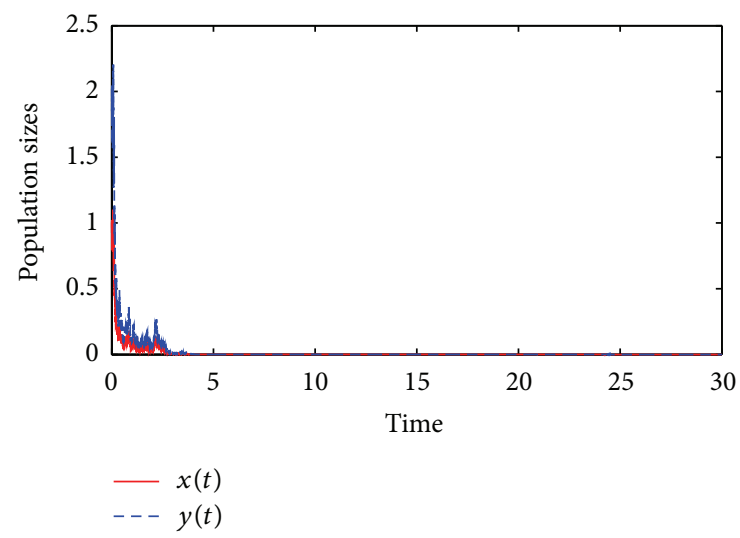

(a)

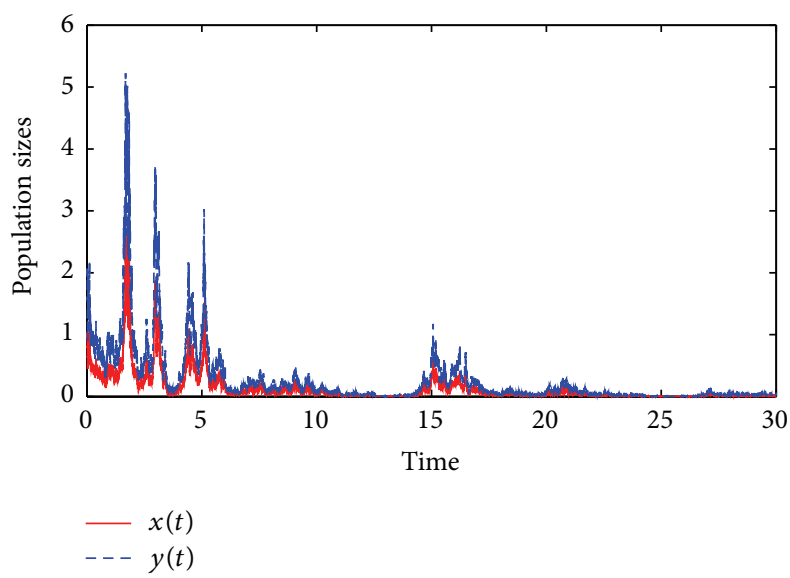

(c)

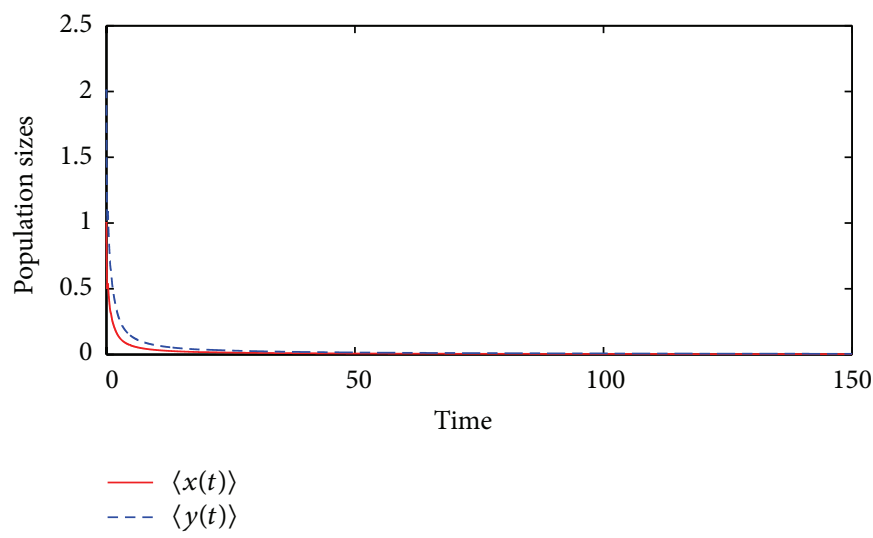

(b)

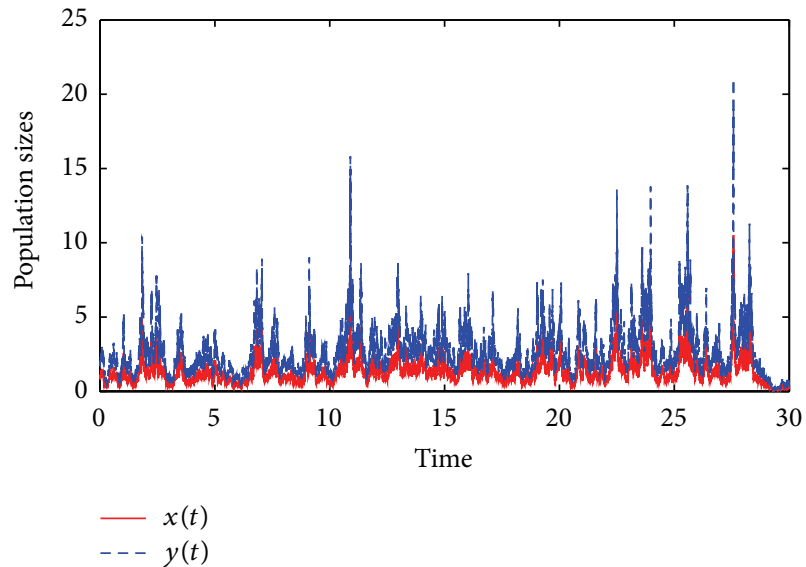

(d)

Figure 1: Persistence and extinction of model (68). (a) Extinction a.s. (b) Nonpersistence in the mean a.s. (c) Weak persistence a.s. (d) Stochastic permanence.

are satisfied. In view of Theorem 4 , the population $x(t)$ and the population $y(t)$ will go to extinction a.s. In Figure 1(b), we consider $r_{i}(t)=1.12505+0.4 \sin t$; the effects of interspecific (for $i \neq j$ ) and intraspecific (for $i=j$ ) interaction at time $t$, that is, $a_{i j}(t)$, are the dominant factor; then the conditions of Theorem 5 hold. By virtue of Theorem 5 , the population $x(t)$ and the population $y(t)$ are nonpersistent in the mean a.s. In Figure 1(c), we choose $r_{i}(t)=1.6+0.4 \sin t ; a_{i j}(t)$ plays the dominant role; then the conditions of Theorem 6 are satisfied. That is to say, the population $x(t)$ and the population $y(t)$ are weak persistence a.s. This means that even though the species $x(t)$ and $y(t)$ are in the process of harvesting, $a_{i j}(t)$ plays the dominant role; then the opportunity of the survival of it still exists. In Figure 1(d), we consider $r_{i}(t)=4.9$; the growth rate is large enough; then the conditions of Theorem 7 hold. Making use of Theorem 7, the population $x(t)$ and the population $y(t)$ are stochastic permanence. By the numerical simulations, we can find that stochastic noise on $r_{i}(t)(1 \leq i \leq n)$ can change the properties of the population models significantly.

\section{Conclusions}

In this paper, the persistence and extinction of a general stochastic nonautonomous $\mathrm{N}$-species Lotka-Volterra model with time-varying, infinite delays and impulsive perturbations are investigated. Sufficient conditions for extinction, nonpersistence in the mean, weak persistence, and stochastic permanence are established in Theorems 4-7. The influences of the stochastic noises and impulsive perturbations on the properties of the stochastic model are discussed. On one hand, if the noise is small enough, the property permanence that the related deterministic system possesses is preserved in the stochastic model. On the other hand, with the increase of noise, the solution of the considered model (2) that will become extinct with probability one, nonpersistent in the mean, or weakly persistent has also been shown in this paper. According to $g_{i}^{*}=\limsup _{t \rightarrow+\infty} t^{-1}\left[\sum_{0<t_{k}<t} \ln (1+\right.$ $\left.\left.h_{i k}\right)+\int_{0}^{t}\left(r_{i}(s)-\sigma_{i}^{2}(s) / 2\right) d s\right]$, we can obtain the result that if the impulsive perturbations are bounded, the properties including extinction, nonpersistence in the mean, weak persistence, and stochastic permanence are not affected by the impulsive perturbations and if the impulsive perturbations are unbounded the properties will be affected by the impulsive perturbations and change greatly. Moreover, the critical number between extinction and weak persistence is obtained. Through the observation of Theorems $4-7$, there is a very interesting phenomenon that the stochastic noise 
on $r_{i}(t)$ is detrimental to the survival of the population but the stochastic noise on $a_{i j}(t)$ has hardly impressed on the persistence or extinction of the population. Finally, the numerical simulations are given to confirm the theoretical analysis results.

\section{Conflict of Interests}

The authors declare that there is no conflict of interests regarding the publication of this paper.

\section{Authors' Contribution}

The work presented here was carried out in collaboration between all authors. Qing Wang and Yongguang Yu conceived and designed the study. Qing Wang and Shuo Zhang performed the experiments, carried out the numerical simulations, analyzed the data, and interpreted the results. Qing Wang wrote the paper. Qing Wang, Yongguang Yu, and Shuo Zhang reviewed and edited the paper. All authors have read and approved the final paper.

\section{Acknowledgment}

This work is supported by the National Nature Science Foundation of China under Grant no. 11371049.

\section{References}

[1] H. X. Hu, K. Wang, and D. Wu, "Permanence and global stability for nonautonomous $\mathrm{N}$-species Lotka-Volterra competitive system with impulses and infinite delays," Journal of Mathematical Analysis and Applications, vol. 377, no. 1, pp. 145-160, 2011.

[2] C. L. Shi, Z. Li, and F. D. Chen, "Extinction in a nonautonomous Lotka-Volterra competitive system with infinite delay and feedback controls," Nonlinear Analysis: Real World Applications, vol. 13, no. 5, pp. 2214-2226, 2012.

[3] Z. Li, M. A. Han, and F. D. Chen, "Influence of feedback controls on an autonomous Lotka-Volterra competitive system with infinite delays," Nonlinear Analysis: Real World Applications, vol. 14, no. 1, pp. 402-413, 2013.

[4] Z. J. Du and Y. S. Lv, "Permanence and almost periodic solution of a Lotka-Volterra model with mutual interference and time delays," Applied Mathematical Modelling, vol. 37, no. 3, pp. 10541068, 2013.

[5] Z. Y. Hou, "On permanence of Lotka-Volterra systems with delays and variable intrinsic growth rates," Nonlinear Analysis: Real World Applications, vol. 14, no. 2, pp. 960-975, 2013.

[6] K. Gopalsamy, "Global asymptotic stability in Volterra's population systems," Journal of Mathematical Biology, vol. 19, no. 2, pp. 157-168, 1984.

[7] Y. Kuang, Delay Differential Equations with Applications in Population Dynamics, Academic Press, Boston, Mass, USA, 1993.

[8] T. C. Gard, "Persistence in stochastic food web models," Bulletin of Mathematical Biology, vol. 46, no. 3, pp. 357-370, 1984.

[9] T. C. Gard, Introduction to Stochastic Differential Equations, Marcel Dekker, New York, NY, USA, 1988.
[10] L. Zhenhai, "Anti-periodic solutions to nonlinear evolution equations," Journal of Functional Analysis, vol. 258, no. 6, pp. 2026-2033, 2010.

[11] R. M. May, Stability and Complexity in Model Ecosystems, Princeton University Press, Princeton, NJ, USA, 2001.

[12] M. Liu and K. Wang, "Global asymptotic stability of a stochastic Lotka-Volterra model with infinite delays," Communications in Nonlinear Science and Numerical Simulation, vol. 17, no. 8, pp. 3115-3123, 2012.

[13] V. Lakshmikantham, D. Bainov, and P. Simeonov, Theory of Impulsive Differential Equations, World Scientific Press, Singapore, 1989.

[14] D. Bainov and P. Simeonov, Impulsive Differential Equations: Periodic Solutions and Applications, Longman, Harlow, UK, 1993.

[15] J. Hou, Z. D. Teng, and S. J. Gao, "Permanence and global stability for nonautonomous N-species Lotka-Valterra competitive system with impulses," Nonlinear Analysis: Real World Applications, vol. 11, no. 3, pp. 1882-1896, 2010.

[16] R. H. Wu, X. L. Zou, and K. Wang, "Asymptotic behavior of a stochastic non-autonomous predator-prey model with impulsive perturbations," Communications in Nonlinear Science and Numerical Simulation, vol. 20, no. 3, pp. 965-974, 2015.

[17] M. Liu and K. Wang, "Asymptotic behavior of a stochastic nonautonomous Lotka-Volterra competitive system with impulsive perturbations," Mathematical and Computer Modelling, vol. 57, no. 3-4, pp. 909-925, 2013.

[18] L. Zhang and Z. D. Teng, "N-species non-autonomous LotkaVolterra competitive systems with delays and impulsive perturbations," Nonlinear Analysis: Real World Applications, vol. 12, no. 6, pp. 3152-3169, 2011.

[19] H. Hu, K. Wang, and D. Wu, "Permanence and global stability for nonautonomous $\mathrm{N}$-species Lotka-Volterra competitive system with impulses and infinite delays," Journal of Mathematical Analysis and Applications, vol. 377, no. 1, pp. 145-160, 2011.

[20] S. C. Gaimradt and L. B. Kats, "Effect of introduced crayfish and mosquitofish on California newts," Conservation Biology, vol. 10, no. 4, pp. 1155-1162, 1996.

[21] S. L. Pimm, H. L. Jones, and J. Diamond, "On the risk of extinction," The American Naturalist, vol.132, no. 6, pp. 757-785, 1988.

[22] H. P. Liu and Z. Ma, "The threshold of survival for system of two species in a polluted environment," Journal of Mathematical Biology, vol. 30, no. 1, pp. 49-61, 1991.

[23] T. G. Hallam and M. Zhien, "Persistence in population models with demographic fluctuations," Journal of Mathematical Biology, vol. 24, no. 3, pp. 327-329, 1986.

[24] C. Lu and X. Ding, "Persistence and extinction of a stochastic logistic model with delays and impulsive perturbation," Acta Mathematica Scientia, vol. 34, no. 5, pp. 1551-1570, 2014.

[25] X. Yang, W. Wang, and J. Shen, "Permanence of a logistic type impulsive equation with infinite delay," Applied Mathematics Letters, vol. 24, no. 4, pp. 420-427, 2011.

[26] M. He, Z. Li, and F. Chen, "Permanence, extinction and global attractivity of the periodic Gilpin-Ayala competition system with impulses," Nonlinear Analysis: Real World Applications, vol. 11, pp. 1537-1551, 2012.

[27] J. Hou, Z. Teng, and S. Gao, "Permanence and global stability for nonautonomous $\mathrm{N}$-species Lotka-Valterra competitive system with impulses," Nonlinear Analysis: Real World Applications, vol. 11, no. 3, pp. 1882-1896, 2010. 
[28] H. J. Jiang and Z. D. Teng, "Boundedness, periodic solutions and global stability for cellular neural networks with variable coefficients and infinite delays," Neurocomputing, vol. 72, no. 1012, pp. 2455-2463, 2009.

[29] M. Liu and K. Wang, "On a stochastic logistic equation with impulsive perturbations," Computers and Mathematics with Applications, vol. 63, no. 5, pp. 871-886, 2012.

[30] X. Mao, Stochastic Differential Equations and Applications, Horwood Publishing, Chichester, UK, 1997.

[31] D. J. Higham, "An algorithmic introduction to numerical simulation of stochastic differential equations," SIAM Review, vol. 43, no. 3, pp. 525-546, 2001.

[32] Y. Song and C. T. H. Baker, "Qualitative behaviour of numerical approximations to Volterra integro-differential equations," Journal of Computational and Applied Mathematics, vol. 172, no. 1, pp. 101-115, 2004. 


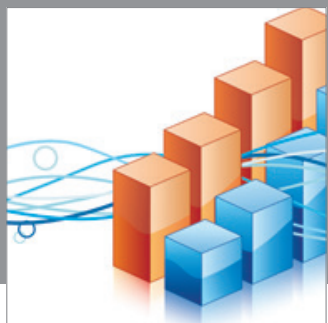

Advances in

Operations Research

mansans

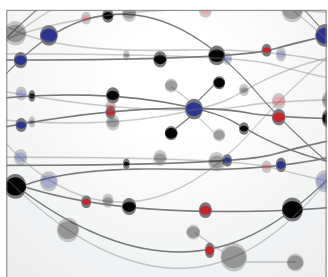

The Scientific World Journal
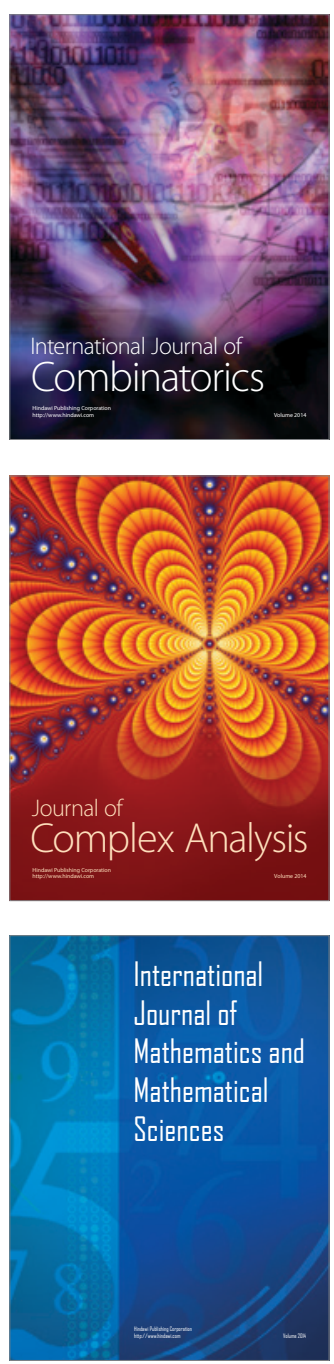
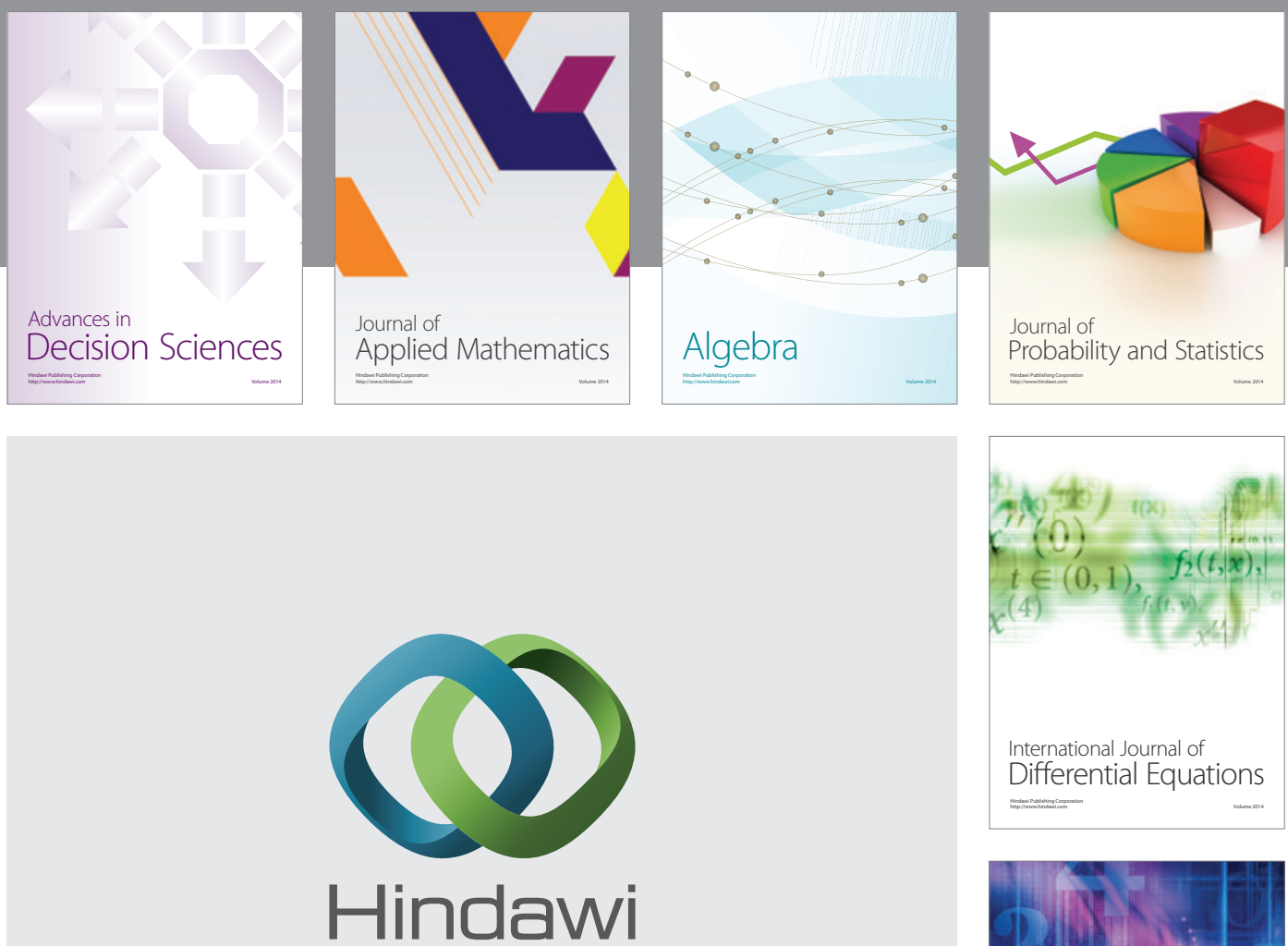

Submit your manuscripts at http://www.hindawi.com
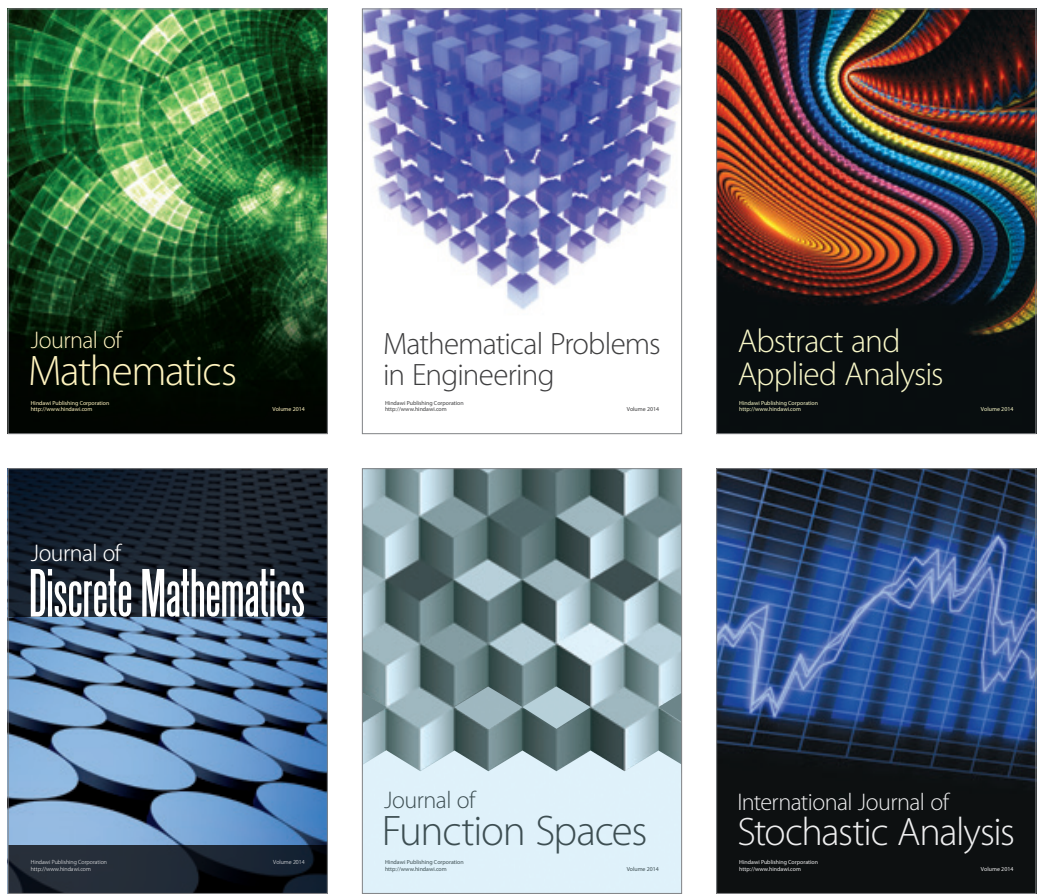

Journal of

Function Spaces

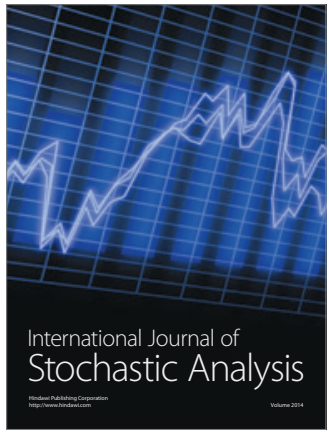

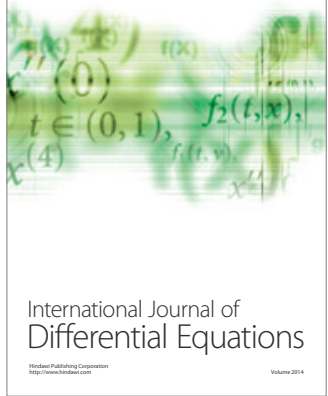
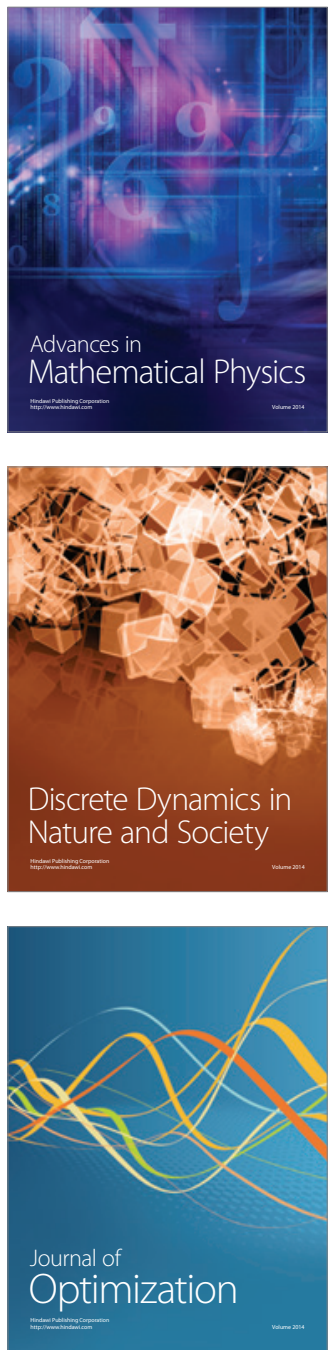